Article

\title{
Symmetric Free Form Building Structures Arranged Regularly on Smooth Surfaces with Polyhedral Nets
}

\author{
Jacek Abramczyk \\ Department of Architectural Design and Engineering Graphics, Rzeszow University of Technology, \\ Al. Powstańców Warszawy 12, 35-959 Rzeszów, Poland; jacabram@prz.edu.pl
}

Received: 21 March 2020; Accepted: 1 May 2020; Published: 6 May 2020

\begin{abstract}
The article is an original insight into interdisciplinary challenges of shaping innovative unconventional complex free form buildings roofed with multi-segment shell structures arranged with using novel parametric regular networks. The roof structures are made up of nominally plane thin-walled folded steel sheets transformed elastically and rationally into spatial shapes. A method is presented for creating such symmetric structures based on the regular spatial polyhedral networks created as a result of a composition of many complete reference tetrahedrons by their common flat sides and straight side edges arranged regularly and symmetrically in the three-dimensional Euclidean space. The use of the regularity and symmetry in the process of shaping different forms of (a) single tetrahedral meshes and whole consistent polyhedral structures, (b) individual plane walls and complex elevations, (c) single transformed folds, entire corrugated shell roofs, and their structures allow a creative search for attractive rational parametric solutions using a few author's parametric algorithms and their implementation as built-in commands of the AutoCAD visual editor or applications of the Rhino/Grasshopper program.
\end{abstract}

Keywords: unconventional building free forms; polyhedral spatial networks; ruled surfaces; transformed corrugated steel roofs; steel shell structures; parametric shaping; visual editor programming; thin-walled folded sheets; symmetric shape transformations

\section{Introduction}

Thin-walled steel sheets are profiled in one direction to use them as members and coverings for roofing. The rationality of using such sheets results from the very favorable ratio of self-weight to load-bearing capacity or covering surface area, and from quick roof assembly [1]. Due to the orthotropic properties of the sheeting, including very different stiffness in two orthogonal directions, flat profiled sheets have been elastically deformed into two shell forms, i.e., rotational cylinder [2,3], Figure 1, and central sectors of right hyperbolic paraboloids [3,4], Figure 2.

Geometric and mechanical changes of the transformed sheets depend on the imposed boundary conditions including the type and degree of the shape transformations. The possibility of using elastically deformed folded sheets as roof coverings depends primarily on the amount of the initial stresses caused by the shape transformations. Therefore, shallow hyperbolic paraboloid sheeting shaped as a result of small twist transformations are most often used [2], Figure 2.

Thin-walled steel sheets having open profiles and folded in one direction can be joined with their longitudinal edges into nominally flat sheeting and transformed into ruled shell shapes as a result of spreading onto at least two skew roof directrices, Figure 3a. The shell shape of each transformed sheeting depends on a mutual position and curvature of two edge directrices. The sheeting can be modeled with a regular smooth ruled undevelopable surface called a warped surface [1], Figure 3b. 


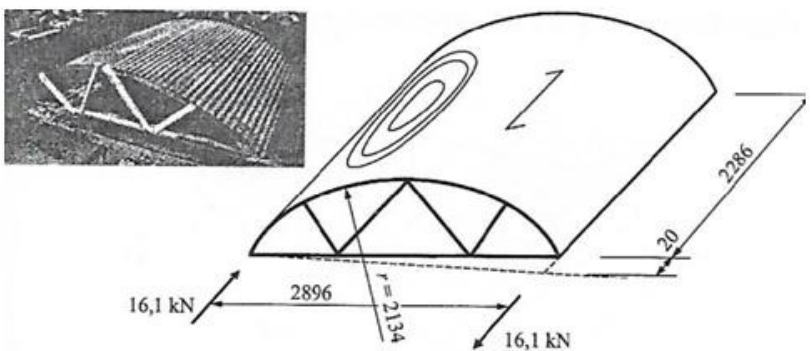

(a)

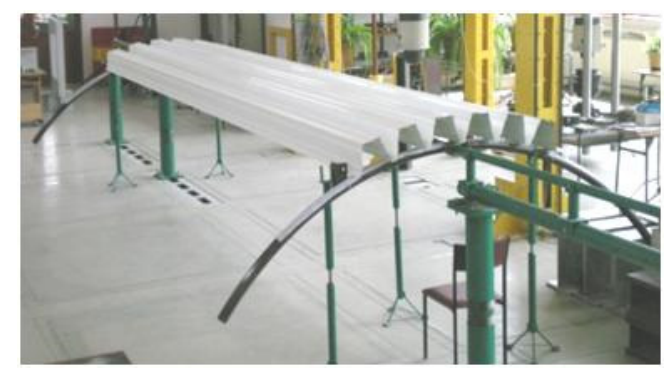

(b)

Figure 1. Elastically bent corrugated cylindrical steel shells: (a) by Abdel_Sayed [2]; (b) by Abramczyk [3].

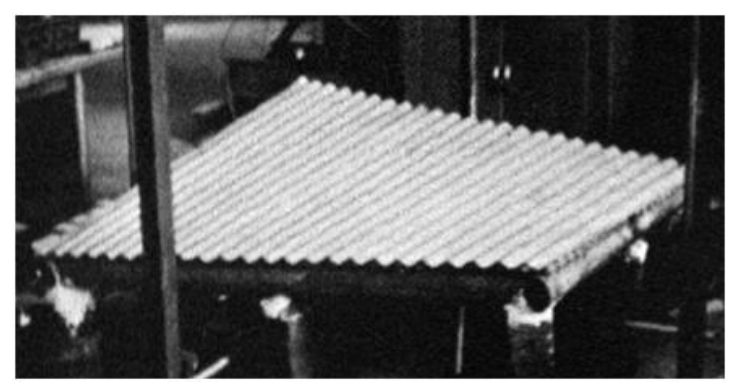

(a)

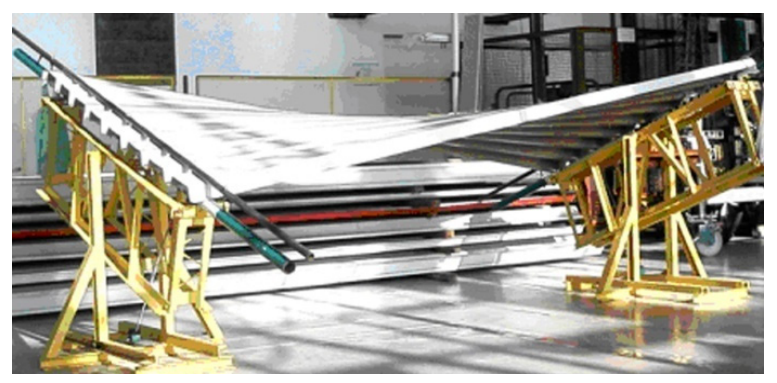

(b)

Figure 2. Elastically twisted hyperbolic paraboloid steel shells: (a) two-layer by J.E. Parker [4]; (b) one-layer by Abramczyk [3].

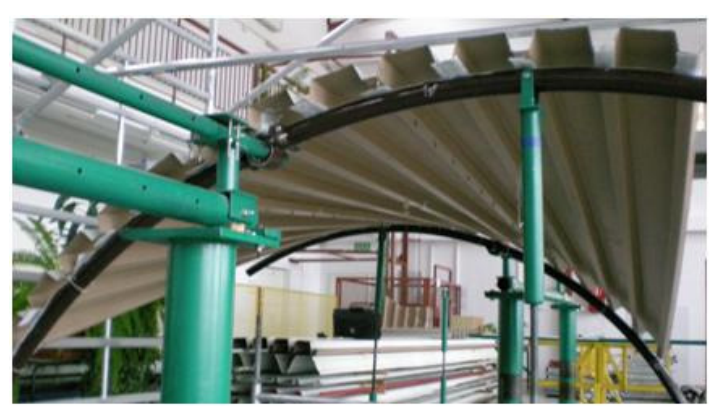

(a)

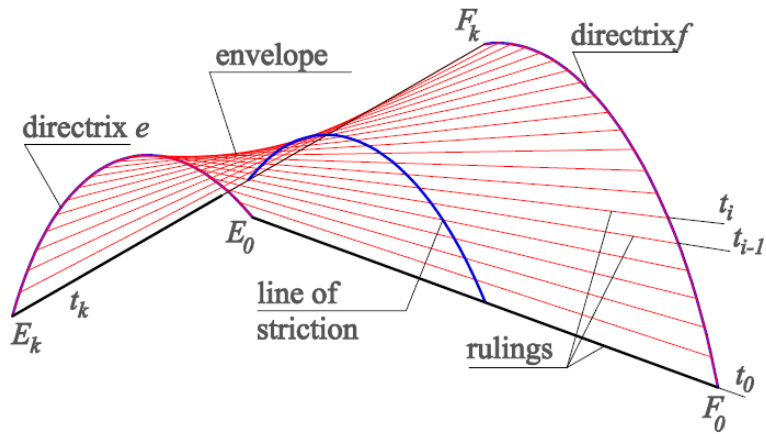

(b)

Figure 3. (a) An axis-symmetric experimental thin-walled corrugated steel shell supported by two curved skew directrices; (b) elements of a smooth model used for shaping the transformed shells.

The analyses related to a static strength work of such deformed and loaded corrugated shells are based on analytical methods leading to calculations of critical forces [5] or FEM describing the entire behavior of these shells [6]. All spatial shape transformations investigated in the present article are effective because freedom of the transverse width increments of each shell fold diversified along its length is ensured [7]. The effective shape transformations are accomplished to obtain a rational static strength work of each shell fold and then very attractive visual building forms [8]. Each shell sheeting transformed effectively, Figure 4, is characterized by a line of contraction passing through the half-length of each shell fold and the smallest possible pre-stresses [9]. 


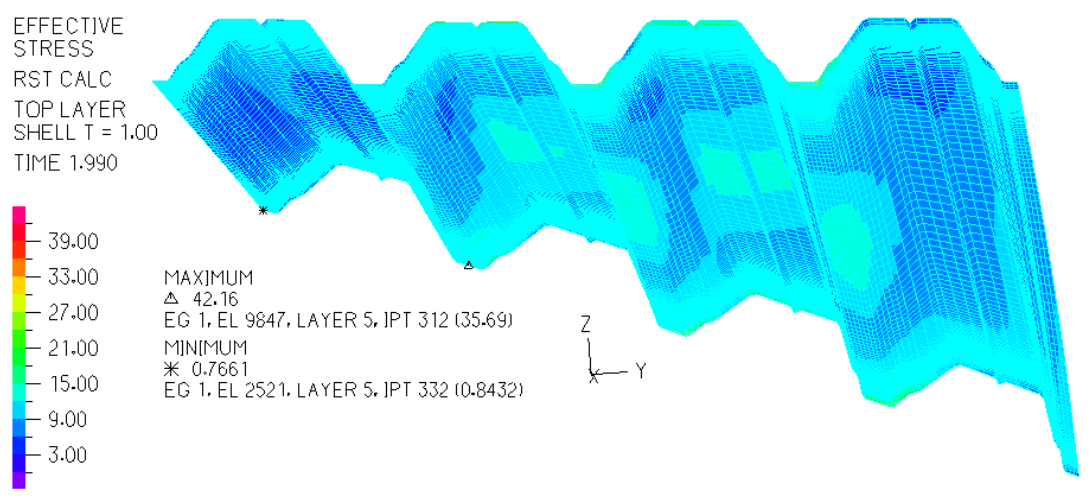

Figure 4. An accurate mechanical thin-walled folded model of a nominally plane folded sheet transformed elastically into a shell shape and the graphical expression of the "effective" stresses in $\mathrm{MPa}$ on its top surface.

The specific feature of the investigated effective shape transformations is that they particularly provide an easy shaping of various symmetric unconventional and rational shell-free forms of roofs, entire buildings, and their structural systems [10], Figure 5a,b. In this way, very attractive free forms of buildings having oblique eaves, girders, and elevations can be shaped [11].

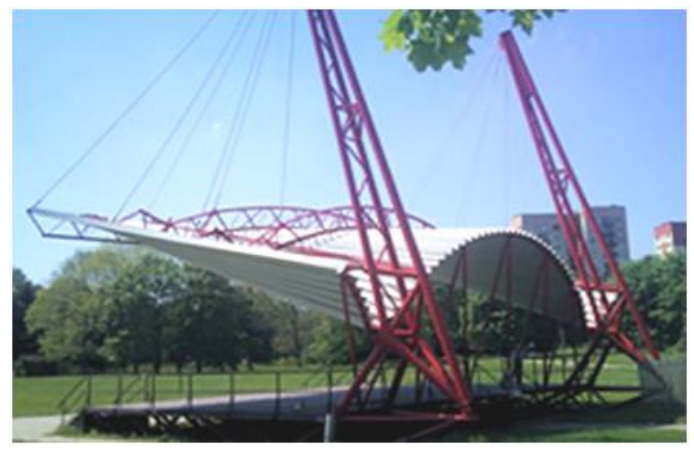

(a)

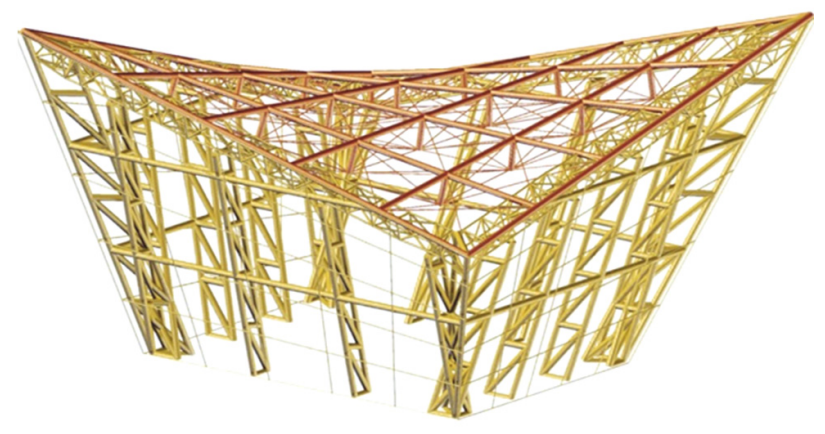

(b)

Figure 5. Two unconventional symmetric shell-free forms: (a) an erected roof shell by Reichhart [10]; (b) a computer model of a bar structural system intended for the transformed shell roof sheeting.

The aforementioned basic properties and restrictions of a rational shaping of single corrugated ruled shell-free forms transformed effectively, concerning the complexity of their shapes, including the contractions, results in the fact that two complete corrugated shell sheets cannot be joined with their crosswise ends, that is perpendicular to their fold's direction, to obtain one resultant smooth shell [3]. Straight or curved edges must appear between two individual shell sheeting joined transversally towards their folds, Figure 6a. Thus, such shells must be modeled by means of complex multi-segment roof shell structures, Figure $6 \mathrm{~b}$. 


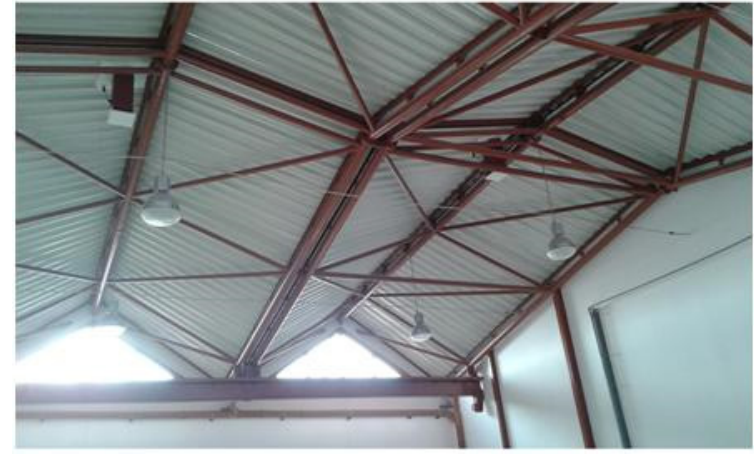

(a)

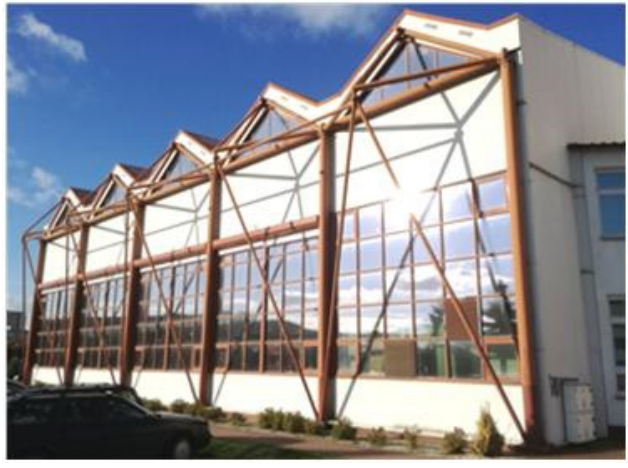

(b)

Figure 6. A symmetric shell structure roofing the experimental hall at Rzeszow University of Technology: (a) an internal view; (b) an external view.

\section{State of the Art}

Thin-walled folded steel sheets of open profiles allow easy deformations of their folds, including their flat rectangular walls and inclination angles between flanges and webs. Nilson studied the possibilities of the sheet's deformations into hyperbolic paraboloid shells and published his research in 1962 [12]. He showed that double-layered fold sheeting transformed elastically into a central sector of a hyperbolic paraboloid or a symmetrical arrangement of four quarters of such a sector is more economical than a reinforced concrete hyperbolic paraboloid shell.

The research conducted under the guidance of Winter [13] confirmed the most important Nilson's conclusions. It was associated with a greater variety of the sheet profiles and dimensions of two-layer hyperbolic paraboloid shells. Central sectors and compositions of quarters of the hyperbolic paraboloid shells were examined. Parker studied roof structures consisting of four folded quarters of a right hyperbolic paraboloid. The analyzed segments were made of two layers of sheets located orthogonally and stiffened with a circumference frame. He analyzed the behavior of the transformed sheeting, including the changes in stiffness and potential energy of these sheets [4]. The Muscat's research [14] concerned primarily critical loads and stability of the sheeting of the type analogous with the one investigated by Parker and Nilsen. Banavalkar made a thorough analysis of the static strength work of these shells [15].

A comprehensive summary of the research performed at Cornwell University is the report made by Gergely et al., [16]. The authors carried out a complete detailed analysis of the static strength work of single and complex profiled hyperbolic paraboloid shells. These shells were made up of plane sheets profiles located in two mutually orthogonal layers, which enables these researches to analyze the shells as isotropic. They examined folded shells of different profiles.

Behavior of a central sector of a folded steel hyperbolic paraboloid stiffened with a circumferential frame was studied by McDermott [17]. Gioncu and Petcu [18] studied the work of the analogous hyperbolic paraboloid shells using traditional analytical analyses of strength and critical loads. They finally developed a novel HYPBUCK computer program for calculating critical loads. They also studied umbrella shell sheeting composed of four symmetrical right hyperbolic paraboloid quarters in various configurations, Figure $7 \mathrm{a}, \mathrm{b}$. 


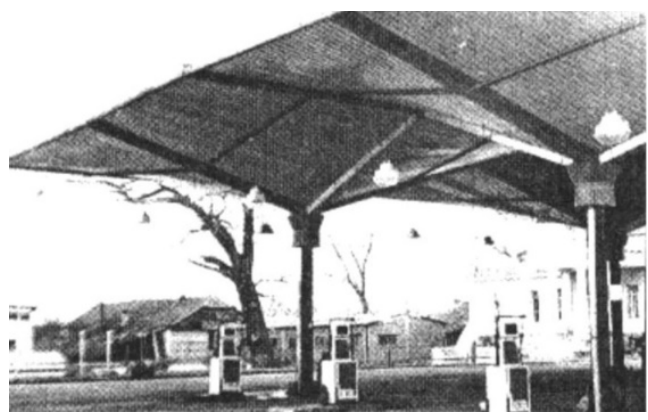

(a)
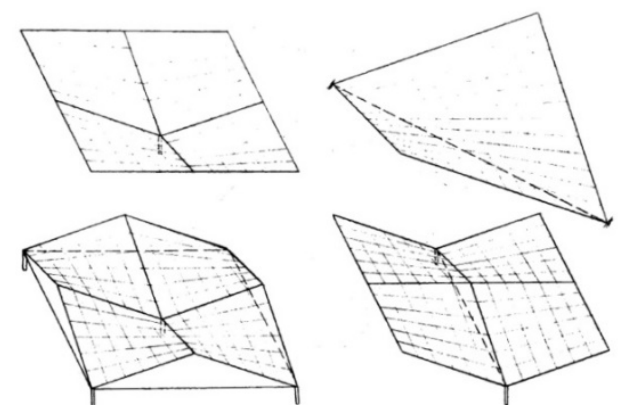

(b)

Figure 7. Symmetrically arranged hyperbolic paraboloid units by Petcu and Gioncu [5]: (a) an erected corrugated umbrella shed; (b) various configurations of umbrella shell structures.

Parallel studies and analyzes related to the static strength work of single and complex hyperbolic paraboloid shells made up of flat folded sheets of different profiles were conducted by Egger et al. [19]. Their method is based on the performed tests, conventional analyses, and analytical calculations of strength and critical loads.

The shells investigated by the aforementioned researchers were undergone forced shape transformations causing relatively big pre-stresses due to the imposed boundary conditions, including the joints between two orthogonal layers arranged over the whole area of the transformed shells and the frames stiffen the quadrangular edges of the shells, so only shallow hyperbolic paraboloid shells called hypars could be created, Figure $8 \mathrm{a}, \mathrm{b}$. In addition, the adjustment of all longitudinal shell fold's axes to the calculated rulings of the designed hyperbolic paraboloid quarters imposes a significant change in the width of the transverse fold's ends passing along each shell directrix affecting important initial stresses. To limit the level of the pre-stresses, a maximum deformation degree has to be introduced. Initial forced deformations of the nominally plane folded sheets have been used by Dawydov in prefabrication of long-span roof panels [20].

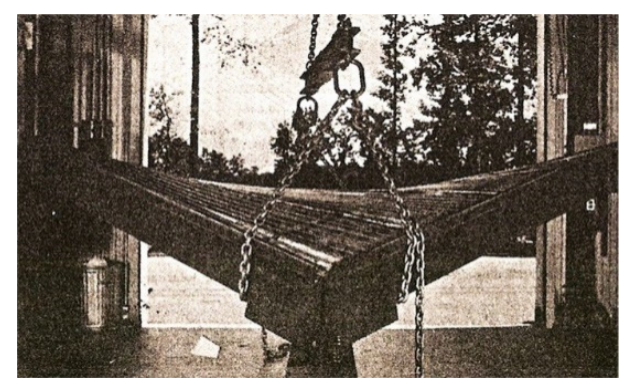

(a)

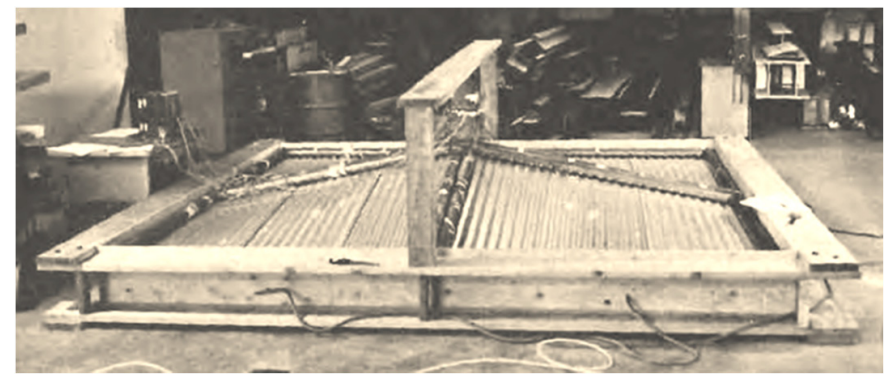

(b)

Figure 8. Two symmetric experimental hyperbolic paraboloid shells: (a) a single shell by McDermott [17]; (b) an umbrella structure of four quarters by Banavalkar and Parker [16].

Davis and Bryan [21] described the most important geometrical and mechanical characteristics of flat and thin-walled transformed shell folds. They presented a complete way of analyzing and designing shells and structures made up of two-layer corrugated sheets located orthogonally. Two most important general conclusions given by these authors and regarding the transformed roof shells are as follows. The researchers found that, theoretically, it is possible to shape many different types of the transformed folded shell sheeting. Practically, however, it is possible to build only cylindrical and hyperbolic paraboloid types of the transformed folded steel shells for roofing due to the available technology.

The use of the well-known conventional design methods $[1,16,19,21]$, known from the traditional courses of theory of thin-walled shells, in shaping of such transformed shell roofs is ineffective because it 
usually results in high values of normal and shear stresses, local buckling and distortion of thin-walled walls: flanges and webs. The assembly of each designed shell sheeting into skewed roof directrices is often impossible because of the plasticity of the fold's edges between flanges and webs. Reichhart developed a specific method for calculating the arrangement and the length of the supporting lines of all folds in transformed one-layer corrugated shell sheeting [10], Figures 5a and 6a,b. The method is based on the orthotropic geometric and mechanic properties of the folded sheets and limits the value of the pre-stresses. His method enables one to shaped right hyperbolic paraboloids or other deep right ruled surfaces [22].

The Reichhart's method is effective only for the cases where the fold's longitudinal axes are perpendicular to roof directrices or very close to those [3]. The method leads to serious errors as it is demonstrated by Abramczyk [3]. These errors result from the lack of conditions providing similar values of stresses at both transverse ends of the same fold. Abramczyk significantly improved the Reichhart's concept and has proposed an innovative method [3,8], so that the transformation would cause the smallest possible initial stresses on the shell folds resulting from this transformation. The visible result of different stress values at both transverse ends of the same shell fold is that the transverse contraction of the fold does not pass halfway along its length, on the contrary, it is shifted closer to one of these ends.

In order to create a method for shaping the considered type of the roof shells transformed rationally, Abramczyk [3] proposed a condition requiring the contraction of each entire shell to pass halfway along the length of each shell fold, Figure $3 a, b$. The condition has to be ensured to obtain a shell fold characterized by the effectiveness of the shape transformations [23]. The Abramczyk's method employs some specific geometric properties of warped surfaces, primarily their lines of striction. The second condition utilized by Abramczyk relates to calculations of the respective surface areas modeling compressing and stretching zones on the transformed folds [24]. Both conditions are based on the results of his experimental tests and computer simulations [25], Figures 1 and 2. They are implemented in the Abramczyk's application [23] developed in the Rhino/Grasshopper program used for parametric modeling of engineering objects.

Simple shell structures composed of a few complete corrugated shells were used in different architectural configurations, most often as shells supported by stiff constructions based on very few columns [24,25]. Such shell structures are used for achieving (a) large spans; (b) greater architectural attractiveness; and (c) skylights letting sunlight into the building interior. Reichhart arranged the complete corrugated shells on horizontal or oblique planes [10] to achieve continuous ribbed structures, Figures 6 and 9 . He developed a simple method for geometrical and strength shaping of the transformed shell roofs. He designed a few corrugated shell sheeting supported by very stiff frameworks or planar girders with additional intermediate directrices, members, and roof bracings.

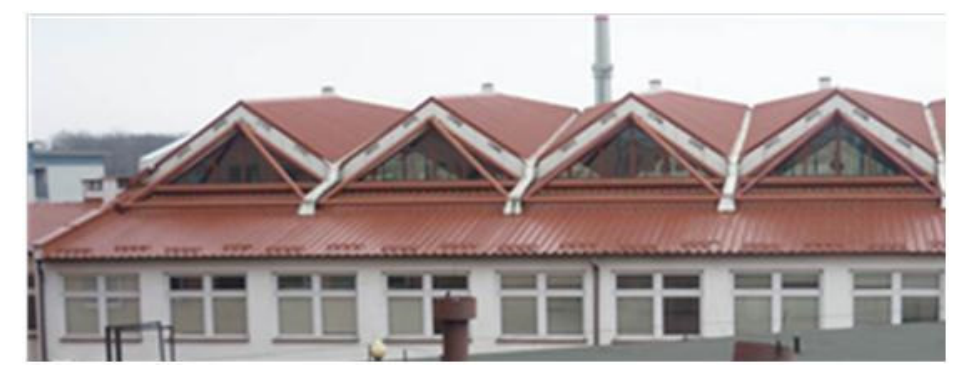

Figure 9. The external view of one elevation of the experimental hall at Rzeszow University of Technology roofed with the shell structure.

In the 70s, Biswas and Iffland [26] presented two concepts of two continuous regular roof structures composed of many identical hyperbolic paraboloid segments made up of transformed folded steel sheets arranged on two various spheres. In the first concept, Figure $10 \mathrm{a}, \mathrm{b}$, they proposed triangular shell segments having three-segment edge lines. Another important feature of this concept is that the proposed plane system, dividing the roof structure into tetrads of triangular shell segments, which is 
based on a sphere. This concept requires significant oblique cuts and big transformation degree of all rectangular folded sheets.

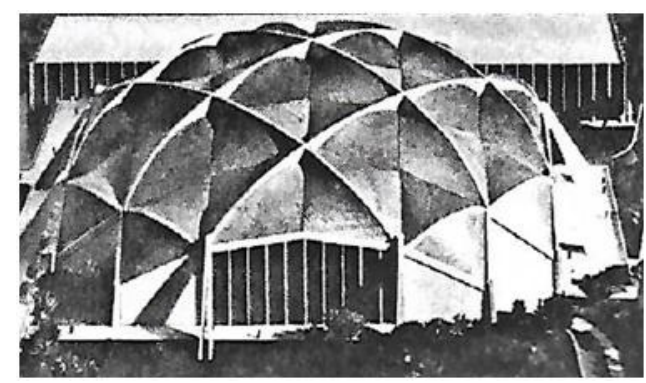

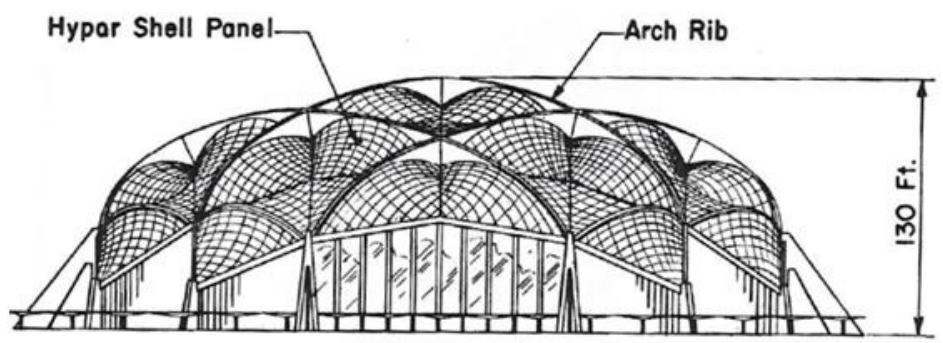

(a)

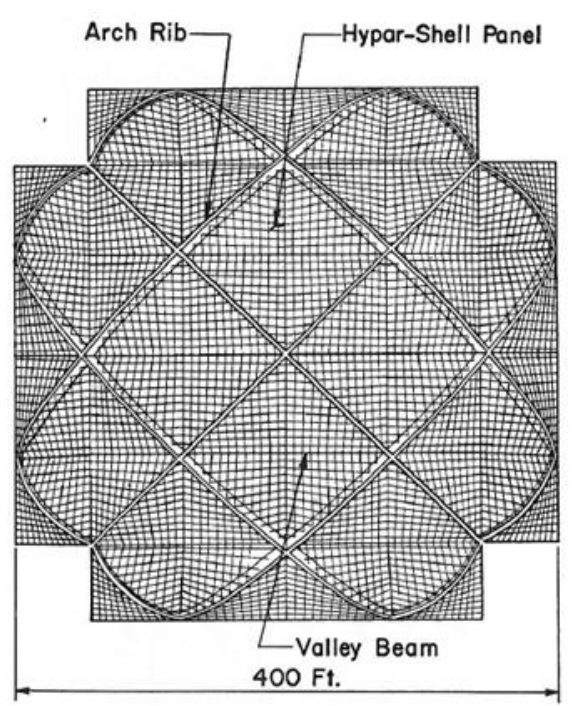

(b)

Figure 10. The first Field House structure proposed by Biswas and Iffland [26]: (a) concept and elevation; (b) plan.

In the second concept, typical quadrilateral transformed hyperbolic paraboloid segments are used, Figure 11a,b. This concept is more realistic, but the degree of twisting and deflection of the complete hyperbolic paraboloid segments are small.

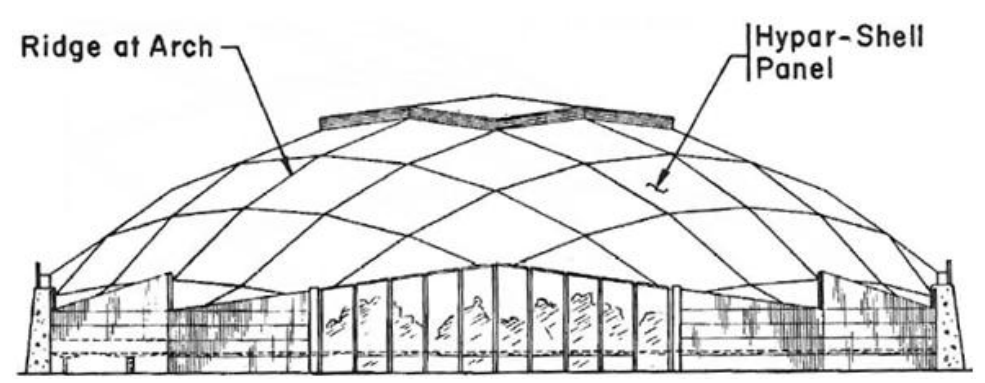

(a)

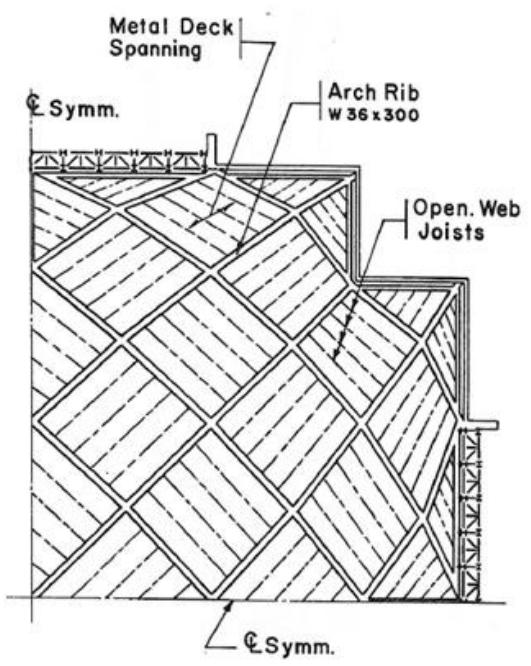

(b)

Figure 11. The second structure proposed by Biswas and Iffland [26]: (a) elevation; (b) framing plan of a quarter of the structure.

At present, shell structures consisting solely of steel decks are not visually appealing. In order to increase their attractiveness, it is possible to use: (1) areas of discontinuity between the metal steel segments, filled with, e.g., glass panels, (2) green plant gardens on the transformed segments, (3) coat the segments with different plastic membranes, (4) communication routes between the segments, (5) a coherent connection of glass facades and steel shell roof. 
In order to create medium and long span free form building structures roofed with complex corrugated shells, Abramczyk $[27,28]$ has proposed certain types of the so-called reference tetrahedrons to model complete free forms covered with folded glass elevations and roofed with complete transformed corrugated steel shell sectors. These tetrahedrons can be arranged regularly in the three-dimensional space to model complex building free forms, Figure 12a,b. Prokopska $[29,30]$ has drawn drew attention to the architectural aspects of shaping such forms.

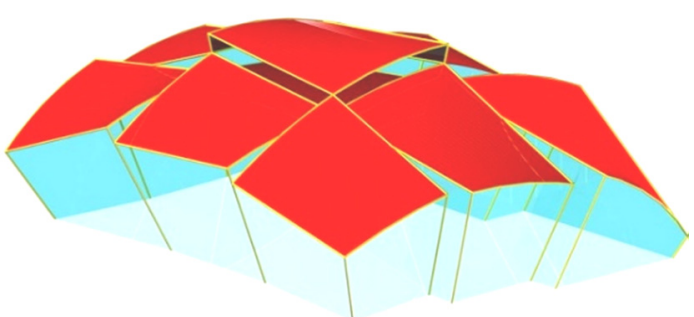

(a)

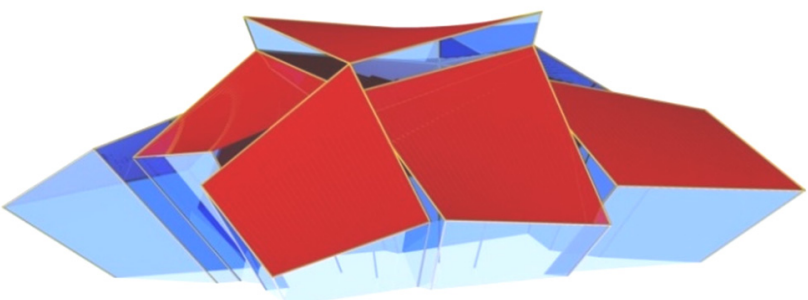

(b)

Figure 12. Two complex building free forms covered with multi-segment roof shell structures and plane-walled folded glass elevations characterized by (a) curved roof directrices; (b) straight roof directrices.

One of the Abramczyk's methods [31] relates to positioning of many aforementioned reference tetrahedrons along ellipses $t_{0}$ and $w_{0}$ contained in two orthogonal principal planes $(x, z)$ and $(y, z)$ of symmetry of a reference ellipsoid $\omega_{r}$, Figure 13a. The method allows the investigated form of a polyhedral structure to be a regular network and precisely take into account by the designer the variable curvature of $\omega_{r}$. The method replaces a finite number of the selected straight lines $t_{i, j}$ normal to $\omega_{r}$ with side edges $k_{i, j}$ of the sought-after reference tetrahedrons. A specific feature of the reference tetrahedrons is that two their subsequent straight side edges $k_{i, j}$ and $k_{i+1, j}$ belonging to the same side must intersect, while two corresponding straight lines $t_{i, j}$ and $t_{i+1, j}$ normal to $\omega_{r}$ do not intersect to each other, Figure $13 \mathrm{~b}$. The positions of $t_{i, j}$ and $t_{i+1, j}$ have to be replaced by $k_{i, j}$ and $k_{i+1, j}$, so that the positions of $k_{i, j}$ and $k_{i+1, j}$ have to be defined based on the geometric properties of $\omega_{r}$. An architectural study of a free form created with the help of the method is presented in Figure 14.

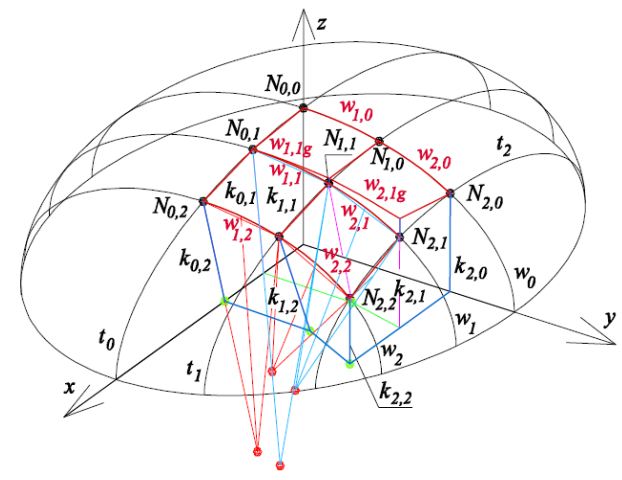

(a)

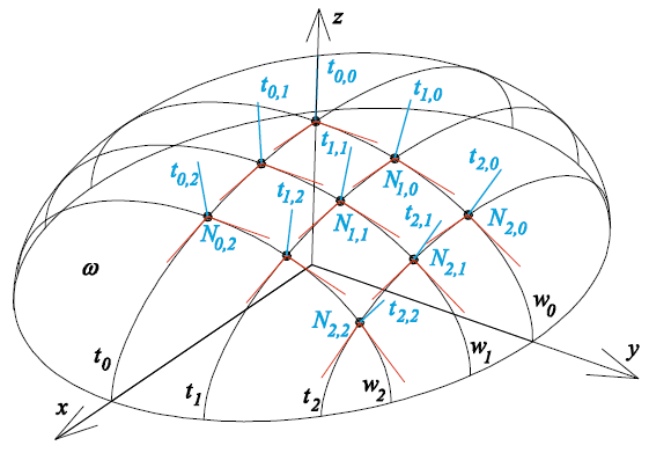

(b)

Figure 13. Creation of a coherent complex free form arranged over a double-curved reference ellipsoid: (a) a quarter of the final structure; (b) normals to the reference ellipsoid.

Parameterization of the reference tetrahedrons enables to computationally search for attractive unconventional building free forms $[23,27]$ and innovative structural systems intended for the investigated complex building free forms. In the analyses of these systems, the supported himself with the following works Obręski [32] developed a few methods for shaping very diversified shell rod 
structures. Rebielak [33] developed steel rod structural systems supporting flat roof covers composed of corrugated sheets. A team of researchers led by Abel and Mungan [34] published comprehensively many examples of the construction systems associated with shaping very diversified roof shells and building free forms. A parametric method for shaping rod shells in the form of Catalan surfaces using the Rhino/Grasshopper program is presented by Dźwierzyńska and Prokopska [35]. The exact geometrical characteristics and methods for determining regular curves and surfaces have been presented by Carmo [36] and Gray [37].

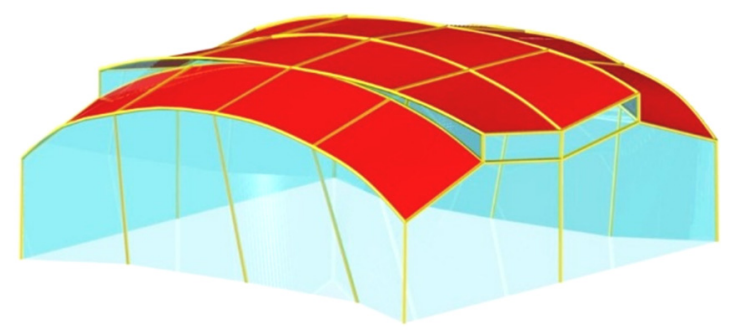

Figure 14. The architectural study of a free form optimized on the basis of the investigated reference ellipsoid.

Transformed folded steel sheets are also used as curved supports for shell panels of individual roof covers [38], Figure 15. Convex roof shells characterized by the positive Gaussian curvature can be created in this way.

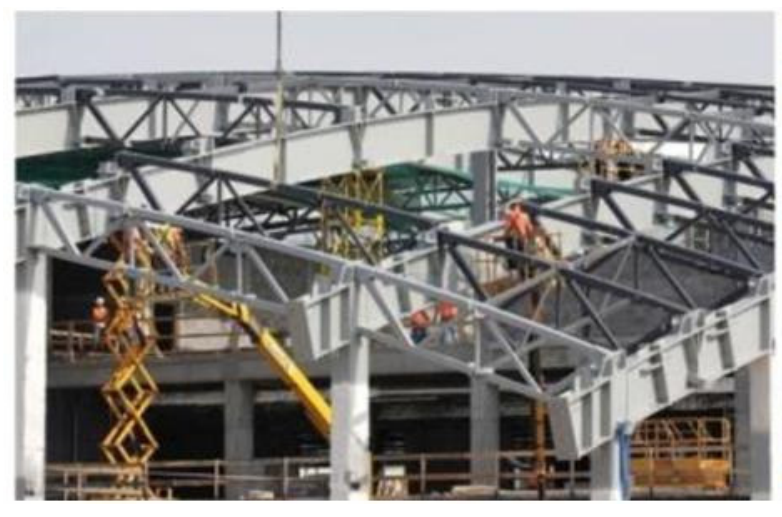

(a)

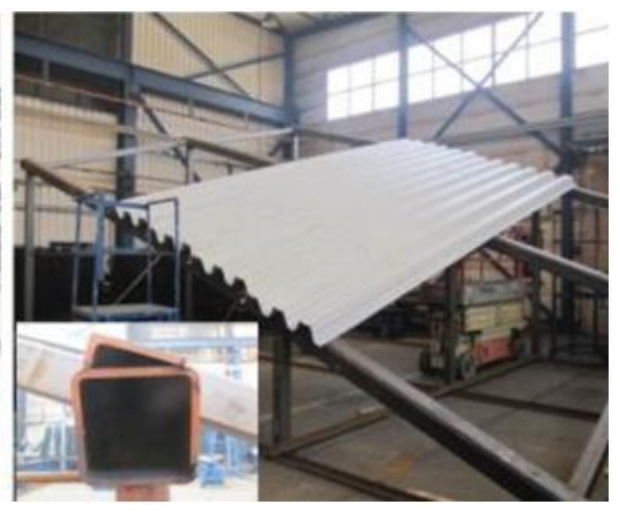

(b)

Figure 15. Two steel structural systems supporting transformed folded sheets by Żwirek [38]: (a) the erection of the final shell roof; (b) experimental tests.

\section{Aim}

The aim is to present a novel method for parametric shaping of building complex free forms based on the innovative spatial polyhedral networks. The presentation is focused on using symmetry for (1) obtaining attractive complex shapes of shell roof structures, folded multi-plane elevations and entire free form buildings, (2) reducing the number of the variables required to define the geometric objects employed, (3) making the proposed algorithm very intuitive, (4) getting rationality of the transformed shell roof forms and their structural systems. The shell roof structures designed with the help of the method are composed of many complete shell sectors arranged in conformity with shapes of various regular reference surfaces. In addition, each elevation should be composed of many planar and oblique walls coherent with the designed shell roof structure. 


\section{Concept of the Method}

The algorithm of the investigated method allows a rational use of the shape transformations of nominally flat thin-walled open folded steel sheets to achieve visually attractive shell roofs whose shapes determine unconventional building free forms as well as their innovative structural systems. Since any two roof directrices are mutually skew straight or curved lines, it is convenient to contain these directrices in the planes of façade walls or in the planes of roof girders. The directrices should be assumed as segments of the roof shell eaves. In addition, a controlled inclination of all elevations to the vertical makes it possible to increase the attractiveness of the created free form buildings.

A smooth resultant shell cannot be the result of a composition of two transformed individual shells with their transverse edges due to the location of the fold's contraction of each effectively transformed roof shell with respect to the roof directrices. Thus, both smooth shells must be separated by a common rib disturbing the smoothness of the resultant complex structure. The ribs- between many complete transformed shells can be taken for common directrices of many pairs of the adjacent shells in the complex roof shell structure.

Following the method's algorithm, a system of the planes separating all roof shell segments and containing the aforementioned edges, including directrices, has to be adopted. Such a spatial system is called a polyhedral reference network $\Gamma$. Each reference network $\Gamma$ is characterized by the following geometrical properties. Each complete mesh $\Gamma_{i j}$ of $\Gamma$ is limited by four adjacent planes of the system defined by means of four vertices $W_{A B i j}, W_{C D i j}, W_{A D i j}$, and $W_{B C i j}$, Figure 16. Each single shell segment $\Omega_{i j}$ and each complete free form $\Sigma_{i j}$ are located in one mesh $\Gamma_{i j}$, so that façade walls, roof directrices and eaves segments are included in the aforementioned quadruple of planes of $\Gamma_{i j}$.

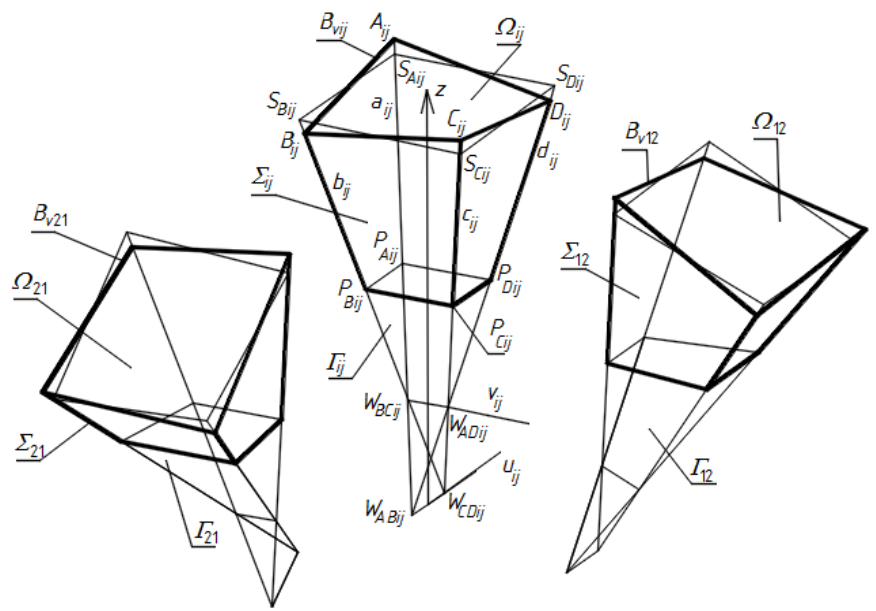

(a)

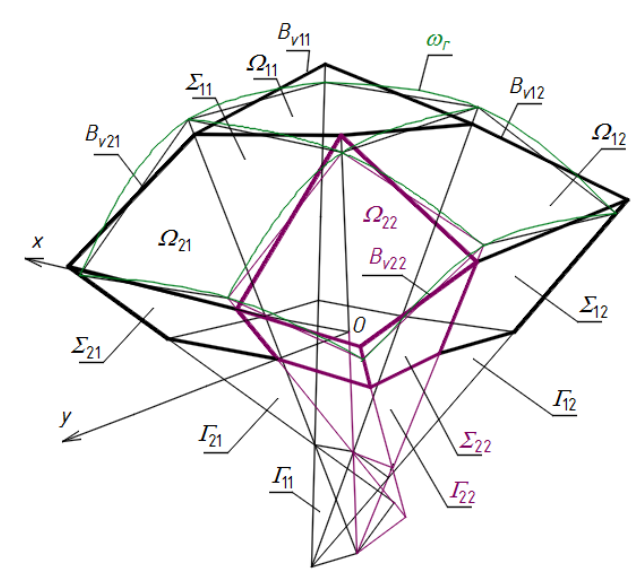

(b)

Figure 16. Creation of a complex free form by means of a reference network $\Gamma$ composed of tetrahedral meshes $\Gamma_{i j}(i=j=1)$ : (a) $\Gamma_{i j}$ before setting $(\mathbf{b}) \Gamma_{i j}$ after setting in $\Gamma$.

Every two adjacent planes of each single mesh $\Gamma_{i j}$ intersect in the side edges: $a_{i j}, b_{i j}, c_{i j}, d_{i j}$, and, every two opposite planes intersect in the axes $u_{i j}$ or $v_{i j}$ of $\Gamma_{i j}$. The side edges and axes of $\Gamma_{i j}$ are defined by means of four vertices $W_{A B i j}, W_{C D i j}, W_{A D i j}, W_{B C i j}$, Figure $16 a$. Thus, each possible triple of these vertices determines one plane of $\Gamma_{i j}$. On the basis of these vertices, four points $S_{A i j}, S_{B i j}, S_{C i j}$ and $S_{D i j}$ are constructed on four side edges $a_{i j}, b_{i j}, c_{i j}, d_{i j}$. These points are vertices of a spatial quadrangle $S_{A i j} S_{B i j} S_{C i j} S_{D i j}$ determining a certain piece of a reference surface $\omega_{r}$, Figure 16b. In relation to $\omega_{r}$, four vertices $A_{i j}, B_{i j}, C_{i j}, D_{i j}$ of single eaves $B_{v i j}$ are determined to obtain mutually skew roof directrices. Vertices $P_{A i j}, P_{B i j}, P_{C i j}, P_{D i j}$, Figure 16a, belonging to a flat horizontal base of the sought-after free form $\Sigma$ are constructed in relation to the aforementioned four vertices $W_{A B i j}, W_{C D i j}, W_{A D i j}, W_{B C i j}$. The complex 
free form $\Sigma$ created on the basis of such a reference network $\Gamma$ is a sum of all individual free forms $\Sigma_{i j}$. Finally, a resultant $z$-axis symmetric free form structure can be achieved, Figure 17a,b.

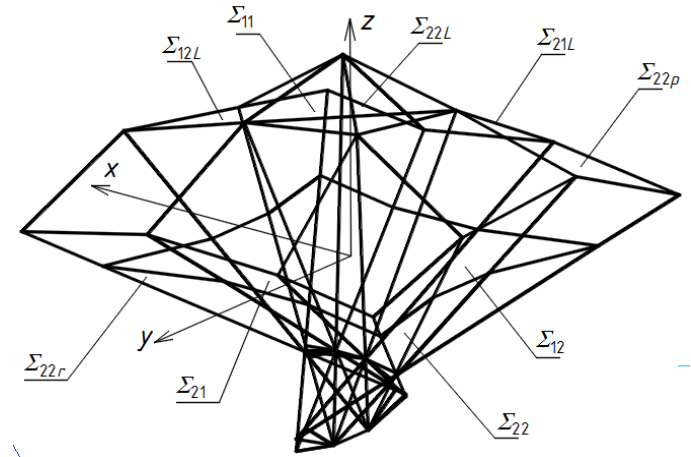

(a)

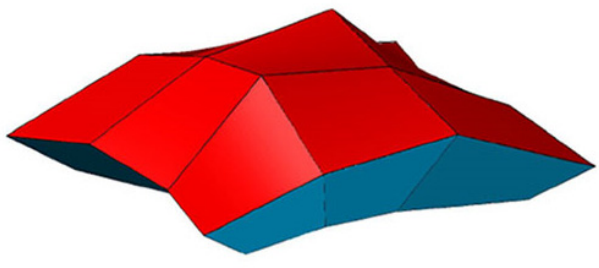

(b)

Figure 17. A complex free form building structure $\Sigma$ roofed with a continuous shell structure: (a) edge model; (b) architectural stadium.

Parameterization realized in the process of the geometric shaping of such free forms $\Sigma$ is based on a definition of a finite set of variables entering into computer application in the form of either the measures of stiff motions, like rotations and translations, or division coefficients of all pairs of the adopted vertices of $\Gamma$. An algorithm of the stiff motions leading to the creation of $\Sigma$ is presented in Section 5. An example of using the division coefficients in creating a spatial reference network is presented in Section 6.

\section{Method's Algorithm}

In the first step of the method's algorithm, the first mesh $\Gamma_{11}$ of a reference network $\Gamma$ is created so that the positions of its four vertices $W_{A B 11}, W_{C D 11}, W_{A D 11}$, and $W_{B C 11}$ are defined in the three-dimensional space. For this purpose, a global coordinate system $[x, y, z]$ must be taken, Figure 18a, where a point $O$ is the origin of $[x, y, z]$. These vertices are arranged symmetrically in relation to the principal planes of $[x, y, z]$, so the sought-after mesh must be symmetric. The first set of initial data is formed from the measures of the vectors and angles employed to determine all characteristic points of $\Gamma_{11}$.

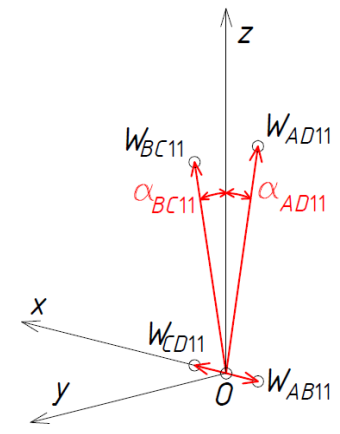

(a)

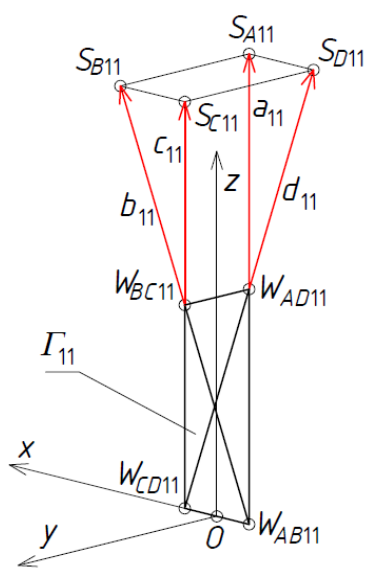

(b)

Figure 18. Creation of $\Gamma_{11}$ : (a) vertices: $W_{C D 11}, W_{A B 11}, W_{A D 11}$ and $W_{B C 11} ;(\mathbf{b})$ side edges: $a_{11}=\left(W_{A D 11}\right.$, $\left.W_{A B 11}\right), b_{11}=\left(W_{B C 11}, W_{A B 11}\right), c_{11}=\left(W_{C D 11}, W_{B C 11}\right)$ and $d_{11}=\left(W_{A D 11}, W_{C D 11}\right)$. 
On the basis of the aforementioned set of initial data, four vertices of $\Gamma_{11}$ are determined as follows. The position of vertex $W_{C D 11}$ is the result of the translation $T_{O C D 11}$ of the point $O$ by the vector $O W_{C D 11}$ whose measure is defined by means of one element of the first initial set. In an analogous way, the position of vertex $W_{A B 11}$ is defined by means of the translation $\mathrm{T}_{O A B 11}$ of the point $O$ by the vector $O W_{A B 11}$ so that its location is on opposite side of $O$ on the $x$-axis.

The position of vertex $W_{A D 11}$ is the result of a composition of the rotation $\mathrm{O}_{C D 11_{-} A B 11}$ of the $z$-axis about the $x$-axis by the angle $\alpha_{A D 11}$ and the translation $\mathrm{T}_{O A D 11}$ of $O$ by the vector $O W_{A D 11}$, where the measures of $\alpha_{A D 11}$ and $O W_{A D 11}$ are two elements of the first set of initial data. The position of vertex $W_{B C 11}$ can be obtained in an analogous way, that is, by means of the rotation $\mathrm{O}_{A B 11} C D 11$ of the $z$-axis about the $x$-axis by the angle $\alpha_{B C 11}$ opposite to $\alpha_{A D 11}$ and the translation $\mathrm{T}_{O B C 11}$ of $O$. If we want to achieve a $z$-axis-symmetric reference tetrahedron, the absolute values of the above vectors and angles must be equal to each other, respectively.

The obtained vertices $W_{A B 11}, W_{C D 11}, W_{A D 11}$ and $W_{B C 11}$ determine four straight side edges: $a_{11}, b_{11}, c_{11}$ and $d_{11}$ of $\Gamma_{11}$. In order to obtain four points $S_{A 11}, S_{B 11}, S_{C 11}$ and $S_{D 11}$ of a reference surface and four vertices $A_{11}, B_{11}, C_{11}$ and $D_{11}$ of eaves $B_{v 11}$ of a shell roof structure, four vectors have to be measured along the side edges $a_{11}, b_{11}, c_{11}$ and $d_{11}$, Figure $19 \mathrm{a}, \mathrm{b}$, so that $S_{A 11}=\mathrm{T}_{S A 11}\left(W_{A D 11}\right)$, $S_{B 11}=\mathrm{T}_{S B 11}\left(W_{B C 11}\right), S_{C 11}=\mathrm{T}_{S C 11}\left(W_{B C 11}\right)$, and $=\mathrm{T}_{S D 11}\left(W_{A D 11}\right)$, and $A_{11}=\mathrm{T}_{A 11}\left(S_{A 11}\right), B_{11}=\mathrm{T}_{B 11}\left(S_{B 11}\right)$, $C_{11}=\mathrm{T}_{C 11}\left(S_{C 11}\right)$, and $D_{11}=\mathrm{T}_{D 11}\left(S_{D 11}\right)$. The measures of these vectors belong to the set of initial data.

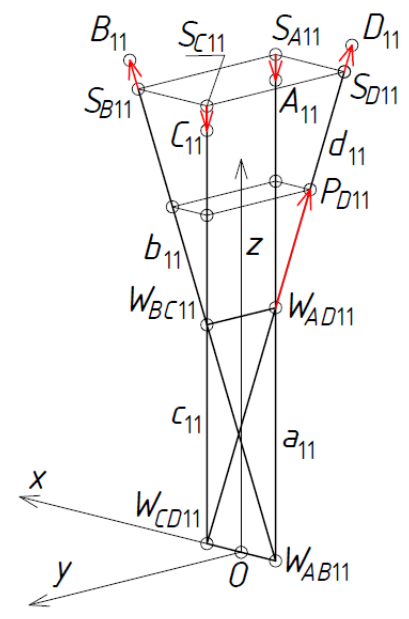

(a)

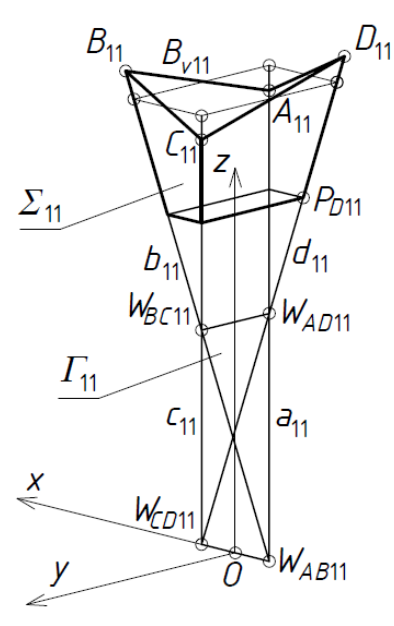

(b)

Figure 19. Two subsequent steps of the creation of $\Sigma_{11}$ : (a) vertices $A_{11}, B_{11}, C_{11}$ and $D_{11} ;(\mathbf{b})$ eaves $B_{v 11}$.

In order to determine a horizontal plane base of the complete free form $\Gamma_{11}$, a point $P_{D 11}=\mathrm{T}_{P D 11}$ $\left(W_{C D 11}\right)$ has to be defined on $d_{11}$. The measure of the vector $W_{C D 11} P_{D 11}$ must be one of the elements of the first set of initial data. Other points of this base can be calculated as the intersection of the horizontal base plane passing through the point $P_{D 11}$ and the four side edges of $\Gamma_{11}$.

The second step of the algorithm relates to the determination of the reference tetrahedron $\Gamma_{12}$, Figure 20a,b. At first, a second set of initial data must be adopted. The set is composed of the measures of the angles and vectors employed in the algorithm to define the positions of all characteristic points of $\Gamma_{12}$.

Four vertices of $\Gamma_{12}$ are determined as follows. Vertex $W_{A B 12}$ is identical with $W_{C D 11}$ of $\Gamma_{11}$ introduced previously. Positions of the vertices $W_{B C 12}=\mathrm{T}_{B C 12}\left(W_{B C 11}\right), W_{A D 12}=\mathrm{T}_{A D 12}\left(W_{A D 11}\right)$ are defined on two side edges $b_{12}=c_{11}, a_{12}=d_{11}$ so that the measures of the vectors $W_{A D 11} W_{A D 12}$ and $W_{B C 11} W_{B C 12}$ are elements of the second set of initial data. The position of vertex $W_{C D 12}$ is obtained as a result of a composition of the rotation $O_{W A D 12 \_W B C 12}$ of $O_{12} W_{A B 11}$ about the axis $W_{A D 12} W_{B C 12}$ by the angle $\alpha_{C D 12}$ and the translation $\mathrm{T}_{C D 12}$ of $O_{12}$ by the vector $O_{12} W_{C D 12}$, where $O_{12}$ is a point of the $\left(W_{A D 12}, W_{B C 12}\right)$ straight line. If the reference tetrahedron $\Gamma_{12}$ is to be symmetrical about the $z$-axis, 
$W_{B C 12}$ and $W_{A D 12}$ have to be symmetric to each other towards the $(x, z)$-plane and $O_{12}$ has to be the middle point of the edge $W_{B C 12} W_{A D 12}$. Four vertices $W_{A B 12}, W_{C D 12}, W_{A D 12}$, and $W_{B C 12}$ determine four straight side edges: $a_{12}, b_{12}, c_{12}$, and $d_{12}$ of $\Gamma_{12}$.

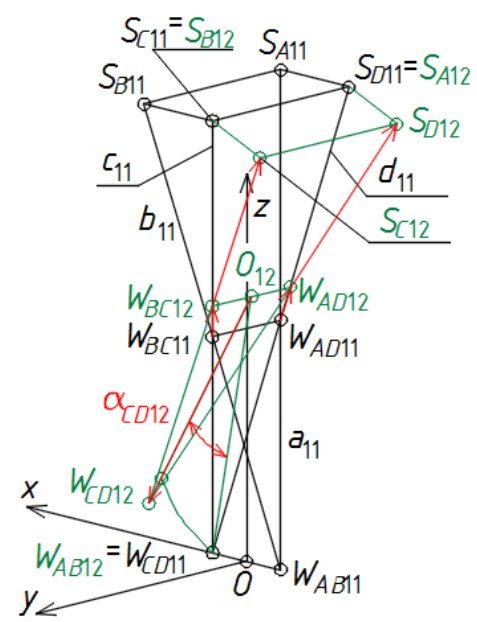

(a)

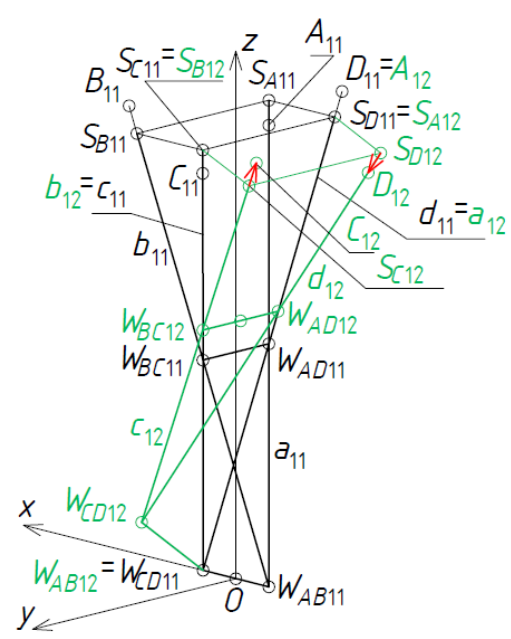

(b)

Figure 20. Two subsequent steps of the determination of (a) the vertices $W_{A B 12}, W_{B C 12}, W_{A D 12}, W_{C D 12}$ of $\Gamma_{12} ;(\mathbf{b})$ the vertices $A_{12}, B_{12}, C_{12}, D_{12}$ of $B_{v 12}$.

Four auxiliary points belonging to a reference surface $\omega_{r}$ and $\Gamma$ are constructed so that $S_{A 12}=S_{D 11}$, $S_{B 12}=S_{C 11}, S_{C 12}=\mathrm{T}_{S C 12}\left(W_{B C 12}\right), S_{D 12}=\mathrm{T}_{S D 12}\left(W_{A D 12}\right)$, where the measures of the vectors $W_{B C 12} S_{C 12}$ and $W_{A D 12} S_{D 12}$ are two elements of the second set of initial data. On the basis of two other elements of the second set, four points $A_{12}=D_{11}, B_{12}=C_{11}, C_{12}=\mathrm{T}_{C 12}\left(S_{C 12}\right), D_{12}=\mathrm{T}_{D 12}\left(S_{D 12}\right)$, Figure 20b, constituting the vertices of a closed spatial quadrilateral line modeling shell roof eaves are constructed.

The third step of the method's algorithm relates to determination of the reference tetrahedron $\Gamma_{21}$, Figure 21a,b. The third set of initial data, composed of the measures of the respective angles and vectors employed to define all specific points of $\Gamma_{21}$ is adopted.

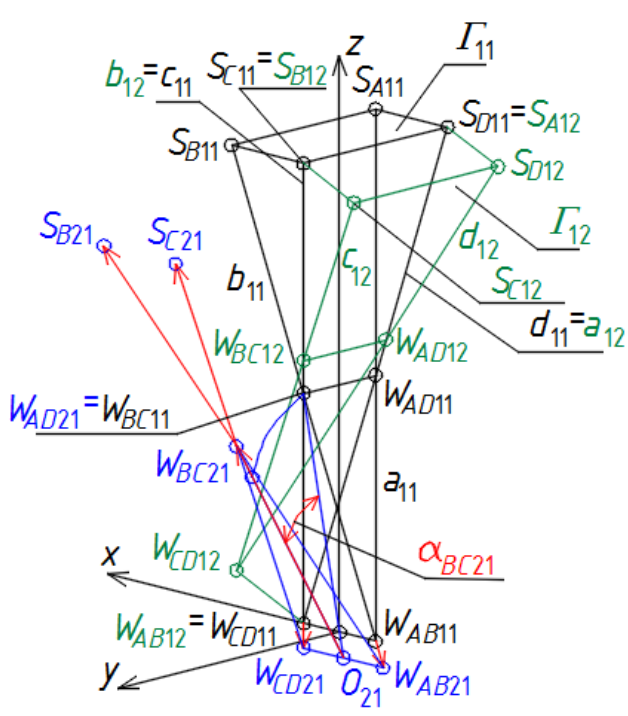

(a)

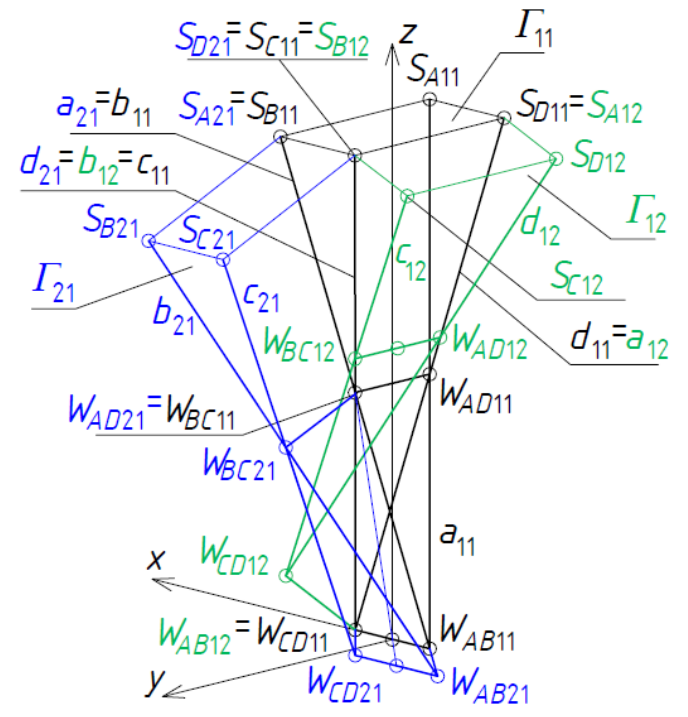

(b)

Figure 21. Two subsequent steps of the method's algorithm related to the construction of; (a) the vertices of $\Gamma_{21} ;(\mathbf{b})$ the eaves side edges of $\Sigma_{21}$. 
Four vertices $W_{A B 21}, W_{C D 21}, W_{A D 21}$, and $W_{B C 21}$ of $\Gamma_{21}$ are determined as follows, Figure 21a. The vertex $W_{A D 21}=W_{B C 11}$. The positions of vertices $W_{A B 21}=\mathrm{T}_{A B 21}\left(W_{A B 11}\right), W_{C D 21}=\mathrm{T}_{C D 21}\left(W_{C D 11}\right)$ are defined on two side edges $a_{21}=b_{11}, d_{21}=c_{11}$, Figure $21 \mathrm{~b}$, so that the measures of the vectors $W_{A B 11} W_{A B 21}$ and $W_{C D 11} W_{C D 21}$ are two elements of the third set of initial data. The position of $W_{B C 21}=\mathrm{T}_{B C 21} \mathrm{O}_{W C D 21 \_W A B 21}\left(W_{B C 11}\right)$ is obtained as a result of a composition of the rotation $\mathrm{O}_{W C D 21 \_W A B 21}$ of $O_{21} W_{A D 21}$ about the axis $W_{C D 21} W_{A B 21}$ by the angle $\alpha_{B C 21}$ and the translation $\mathrm{T}_{B C 21}$ of $O_{21}$ by the vector $O_{21} W_{B C 21}$, where $O_{21}$ is a point of the straight line $W_{C D 21} W_{A B 21}$. If the reference tetrahedron $\Gamma_{21}$ is to be symmetrical towards the $(y, z)$-plane, the positions of points $W_{C D 21}$ and $W_{A B 21}$ have to be symmetric to each other towards the $(y, z)$-plane and $O_{21}$ has to be the middle point of the segment $W_{C D 21} W_{A B 21}$. Four vertices $W_{A B 21}, W_{C D 21}, W_{A D 21}$, and $W_{B C 21}$ determine four straight side edges: $a_{21}, b_{21}, c_{21}$ and $d_{21}$ of $\Gamma_{21}$.

Four auxiliary points belonging to $\omega_{r}$ are determined on the side edges of $\Gamma_{21}$ so that $S_{A 21}=S_{B 11}$, $S_{D 21}=S_{C 11}, S_{C 21}=\mathrm{T}_{S C 21}\left(W_{B C 21}\right)$ and $S_{B 21}=\mathrm{T}_{S B 21}\left(W_{B C 21}\right)$, where the measures of the vectors $W_{B C 21} S_{C 21}$ and $W_{B C 21} S_{B 21}$ belong to the third set of initial data.

The fourth step of the method's algorithm concerns a determination of the reference tetrahedron $\Gamma_{22}$, Figure 22a,b. The fourth set composed of the measures of the vectors and angles employed in this step is adopted.

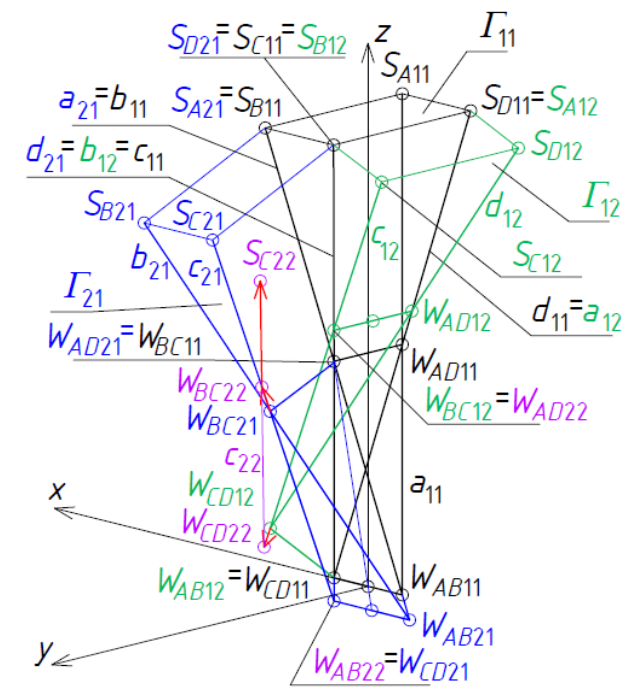

(a)

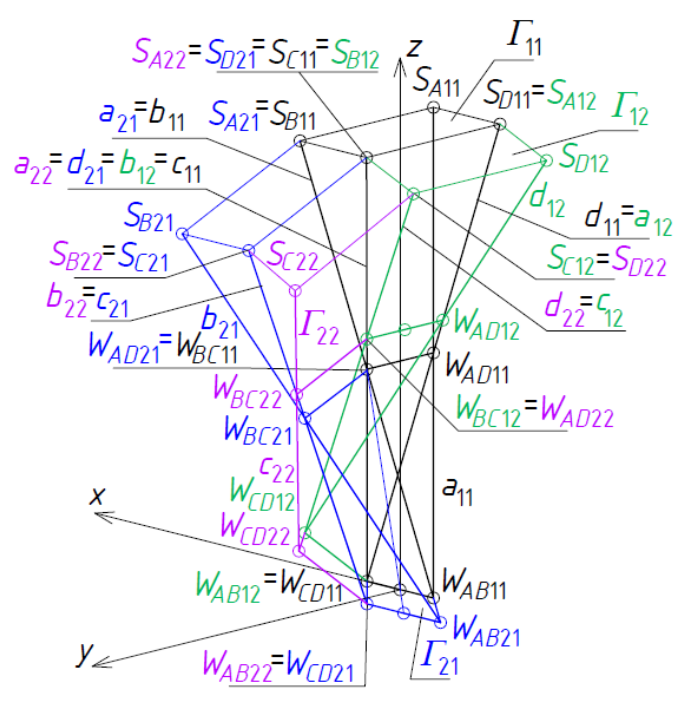

(b)

Figure 22. Two subsequent steps of the method's algorithm related to the construction of (a) all vertices of $\Gamma_{22} ;(\mathbf{b})$ all side edges of $\Sigma_{22}$.

Four vertices $W_{A D 22}=W_{B C 12}, W_{A B 22}=W_{C D 21}, W_{B C 22}=T_{B C 22}\left(W_{B C 21}\right), W_{C D 22}=T_{C D 22}\left(W_{C D 12}\right)$ of $\Gamma_{12}$, Figure 22a, are defined so that the measures of the vectors $W_{B C 21} W_{B C 22}$ and $W_{C D 21} W_{C D 22}$ are two elements of the fourth set of initial data. Four vertices $W_{A B 22}, W_{C D 22}, W_{A D 22}$, and $W_{B C 22}$ determine four straight side edges: $a_{22}, b_{22}, c_{22}$, and $d_{22}$ and two axes $u_{22}$ and $v_{22}$ of $\Gamma_{22}$ Figure 23a.

Four auxiliary points of $\Gamma$ belonging to $\omega_{r}$ are constructed so that $S_{A 22}=S_{C 11}, S_{D 22}=S_{C 12}, S_{B 22}$ $=S_{C 21}$, and $S_{C 22}=T_{C 22}\left(W_{B C 22}\right)$, Figure $23 \mathrm{~b}$, where the measure of the vector $W_{B C 22} S_{C 22}$ is a value belonging to the fourth set of the initial data. On the basis of these points of $\omega_{r}$, four points $A_{22}=C_{11}$, $B_{22}=C_{21}, C_{22}=\mathrm{T}_{\mathrm{C} 22}\left(S_{\mathrm{C} 22}\right), D_{22}=\mathrm{C}_{12}$ constituting the vertices of a closed spatial quadrilateral line modeling complete shell roof eaves are constructed.

The result of adding up the four reference tetrahedrons $\Gamma_{i j}$ constructed above is a subnet $\Gamma_{1}$ constituting about one-fourth of the designed reference network $\Gamma$. The other three parts of $\Gamma$ can be built using $z$-axis symmetry and two $(x, z)$-and $(y, z)$-plane symmetries called 3D-mirrors, Figure 24a,b, in the way described Section 6 with the help of a certain example. All obtained points $S_{A i j}, S_{B i j}$, $S_{C i j}$, 
and $S_{D i j}$, Figure 24a, and their images obtained as a result of the aforementioned symmetries are the selected vertices of a certain net defining $\omega_{r}$, Figure $24 \mathrm{~b}$. In relation to this net, a roof structure $\Omega$ composed of nine sectors $\Omega_{i j}$ is positioned. Thus, the vertices of the eaves of each $\Omega_{i j}$ segment of the roof structure $\Omega$ are defined on the basis of $\omega_{r}$, Figure 25 .

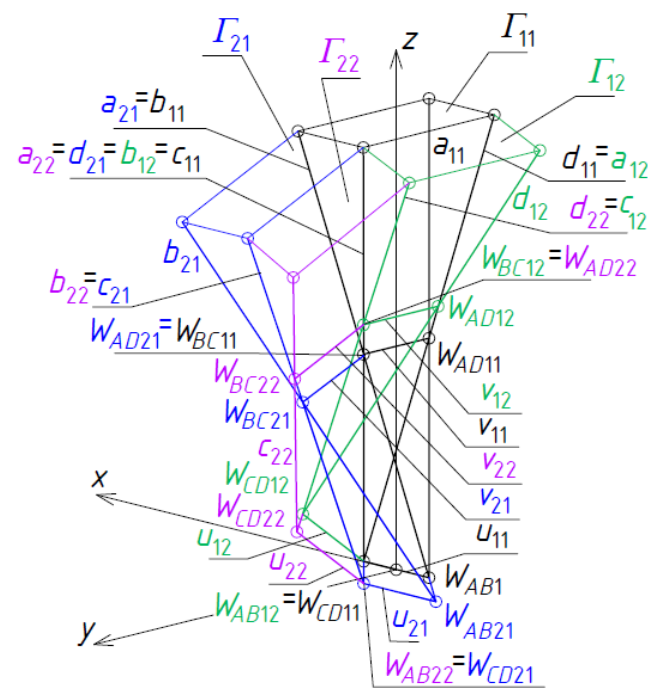

(a)

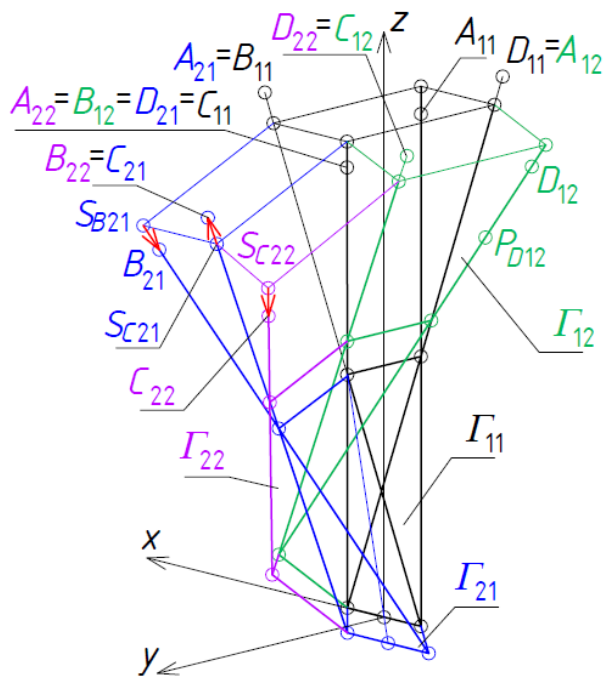

(b)

Figure 23. Subsequent steps of the method's algorithm concerning: (a) definition of the $\Gamma$ 's axes; (b) creation of the vertices of $\Sigma_{21}, \Sigma_{22}$.

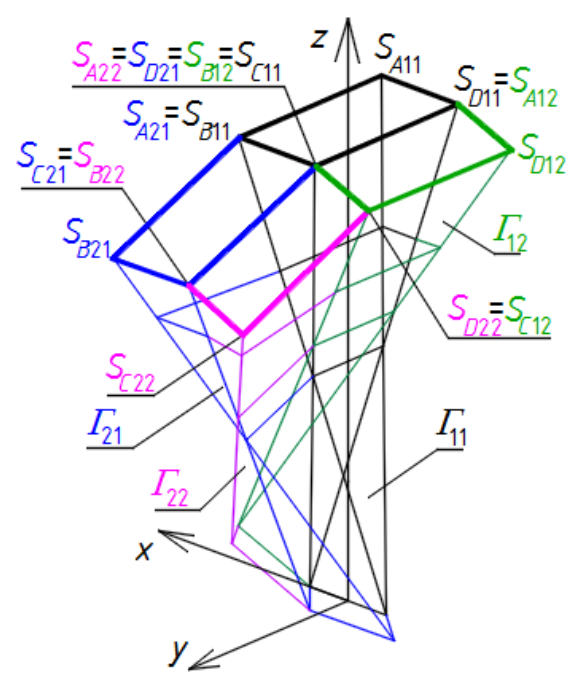

(a)

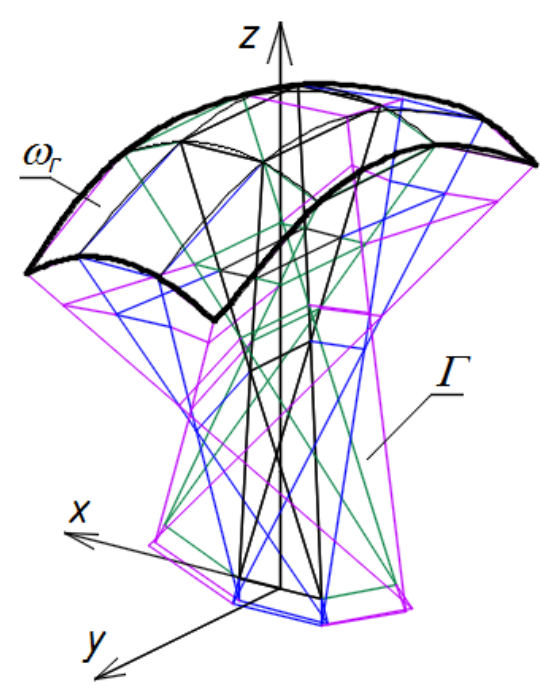

(b)

Figure 24. Subsequent steps of the method's algorithm related to the creation: (a) a quarter of $\Gamma$; (b) the entire network $\Gamma$ and the reference surface $\omega_{r}$ symmetrical about the $z$-axis. 


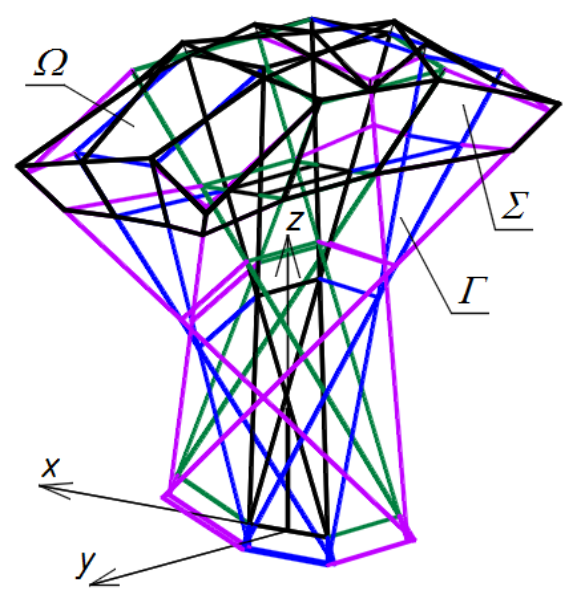

Figure 25. The final step of creating $\Sigma$ and $\Omega$ symmetrical about the $z$-axis.

In addition, it is worth paying attention to the following properties of the reference network $\Gamma$ built so far. The vertices of each reference tetrahedron $\Gamma_{i j}$ designate side edges $a_{i j}, b_{i j}, c_{i j}$, and $d_{i j}$ and planes of $\Gamma$. Each new reference tetrahedron $\Gamma_{i+1 j}$ or $\Gamma_{i j+1}$ is created as a spatial mesh having four sought-after vertices defined in the selected side edges of the previously constructed tetrahedrons $\Gamma_{i j}$ to obtain subsequent pairs of the adjacent meshes having common planes. In the aforementioned planes of $\Gamma$, the locations of roof directrices are determined.

In the case of creating a reference tetrahedron contained in any of the two orthogonal directions related to the first $z$-axially symmetrical tetrahedron $\Gamma_{11}$, one its vertex is laid outside the side edges of the already created subnet of $\Gamma$. This vertex determines a new plane of $\Gamma$ passing through the already constructed axis of this tetrahedron. However, in the case of two directions diagonal in relation to the first $\Gamma_{11}$, each new reference tetrahedron has to have two vertices identical to two from the four previously constructed vertices of $\Gamma$ and two other new vertices have to be determined on two side edges of the previously created subnet of $\Gamma$.

This way of constructing the subsequent reference tetrahedrons located in these orthogonal and diagonal directions leads to the fact that each inner side edge of $\Gamma$ is shared by four adjacent reference tetrahedrons and eight vertices of these four tetrahedrons belong to this side edge. In a general case, these vertices occupy four different positions, in pairs.

Therefore, it seems rational to carry out a process of a geometric parameterization of this type of reference networks, especially the mutual positions of their vertices and other characteristic points. An example of using such an algorithm for a parametric determination of reference polyhedral networks $\Gamma$ and eaves nets $B_{v}$ is given in Section 6, where certain division coefficients of the selected pairs of some adjacent vertices by other vertices of the reference network are employed. In addition, some points belonging to a reference surface and vertices of the eaves edge line of each individual roof shell segment are defined at each side edge of the network $\Gamma$. It is also advisable to use analogous division coefficients of pairs of the reference network's vertices in determining these points of the reference surface and the eaves limiting the individual roof shell segments. An example of making such a parameterization is included in Section 6.

\section{Parametric Shaping of an Example Reference Network and a Free Form Shell Roof}

The algorithm assisted computationally leads to creation of intuitive engineering parametric and regular models of attractive and rational building free forms. To create such models stiff motions including translations and rotations of points and planes presented in the previous section are going to be replaced by slightly more complex actions related to the division coefficients and proportions of some elements of the reference $\Gamma$ and eaves $B_{v}$ networks. These coefficients and proportions allow us to define the positions of (1) the sought-after vertices of $\Gamma$ with respect to the adopted or calculated 
at one of the previous steps pairs of other vertices of $\Gamma_{,}(2)$ the subsequent planes of $\Gamma$, (3) the points $S_{A i r}, S_{B i j}, S_{C i j}$, and $S_{D i j}$ belonging to a reference surface $w_{r},(4)$ the vertices $A_{i j} B_{i j}, C_{i j}$ and $D_{i j}$ of $B_{v}$ relative to the already determined vertices of $\Gamma$ and these points of $w_{r}$.

A use of the method for determining a quarter $\Gamma_{1}$ of a reference network $\Gamma$, Figure 26, and a quarter $B_{v 1}$ of $B_{v}$ consisting of closed spatial quadrangles $B_{v i j}$, is presented below. It is based on some adopted proportions. All vertices of the other three quarters $\Gamma_{2 L}, \Gamma_{3 p}, \Gamma_{4 r}$ of $\Gamma$, Figure 26, and $B_{v 2 L}, B_{v 3 p}, B_{v 4 r}$ of $B_{v}$ are determined using: (1) a $z$-axial symmetry, in the case of $\Gamma_{2 L},(2)$ a $(x, z)$-plane symmetry called 3D-mirror, where $\Gamma_{3 p}$ is constructed, (3) a $(y, z)$-plane symmetry for $\Gamma_{4 r}$.

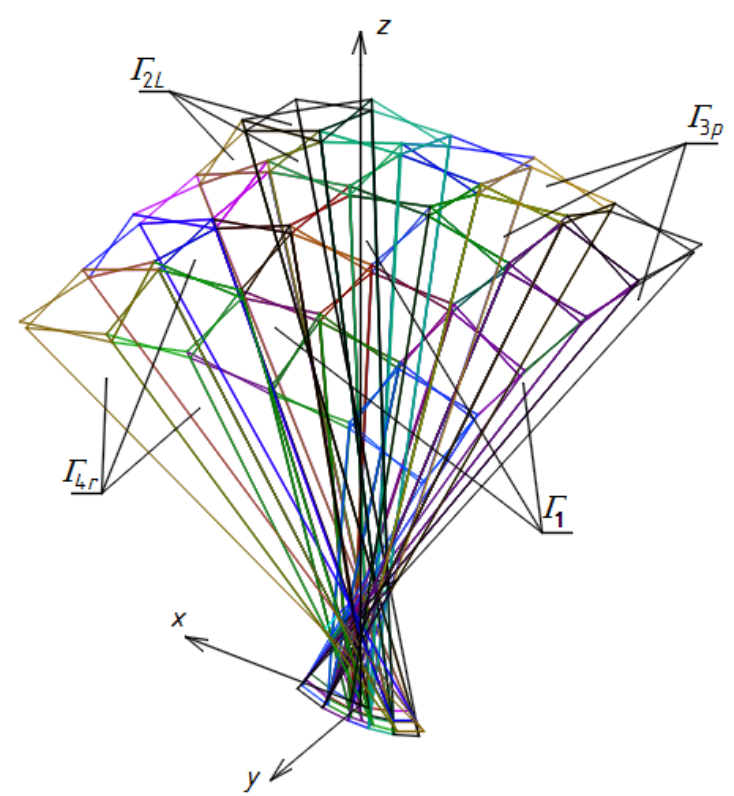

Figure 26. A multi-segment reference network $\Gamma$ composed of four symmetric subnets $\Gamma_{1}, \Gamma_{2 L}, \Gamma_{3 p}$, and $\Gamma_{4 r}$.

Creating the z-axially symmetric network $\Gamma$ is started with defining the first $z$-axially symmetric mesh $\Gamma_{11}$, Figure 27. The subsequent meshes $\Gamma_{i j}$ are determined in the order presented in the previous section, Figures 18-25. In order to build the mesh $\Gamma_{11}$, the following quantities and proportions are adopted. The values of these variables are given in Table 1.

Two opposite planes $\left(W_{A B 11}, W_{C D 11}, W_{B C 11}\right)$ and $\left(W_{A B 11}, W_{C D 11}, W_{A D 11}\right)$, Figure $27 \mathrm{a}-\mathrm{c}$, are inclined to each other at an angle $\alpha_{11}=2 \alpha_{B C 11}=2 \alpha_{A D 11}$, Figure 19. The length of the edge $W_{C D 11} W_{A B 11}$ contained in the $u_{1}$-axis was adopted in accordance with the values given in Table 1 .

Table 1. The initial data defining the shapes of the meshes $\Gamma_{11}$ and $B_{v 11}$ of $\Gamma$ and $B_{v}$.

\begin{tabular}{ccc}
\hline Variable or Division Coefficient & Value & Unit \\
\hline$\alpha_{11}$ & 10.0 & \\
\hline$W_{A B 11} W_{C D 11}$ & 2000.0 & $\mathrm{~mm}$ \\
$O W_{B C 11}$ & $100,00.0$ & $\mathrm{~mm}$ \\
$\mathrm{~d}_{O W 11}$ & 1.0 & - \\
$\mathrm{d}_{S 11}$ & 2.5 & - \\
$\mathrm{dd}_{11}$ & 0.1 & - \\
\hline
\end{tabular}




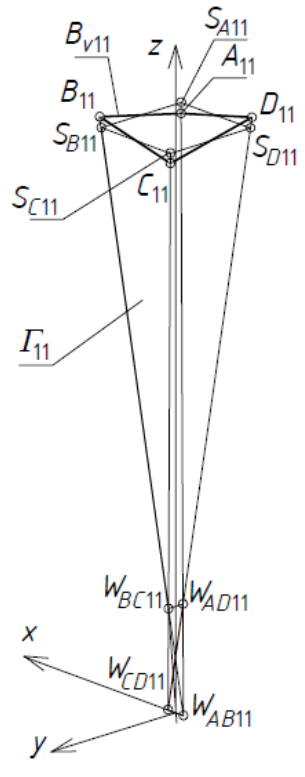

(a)

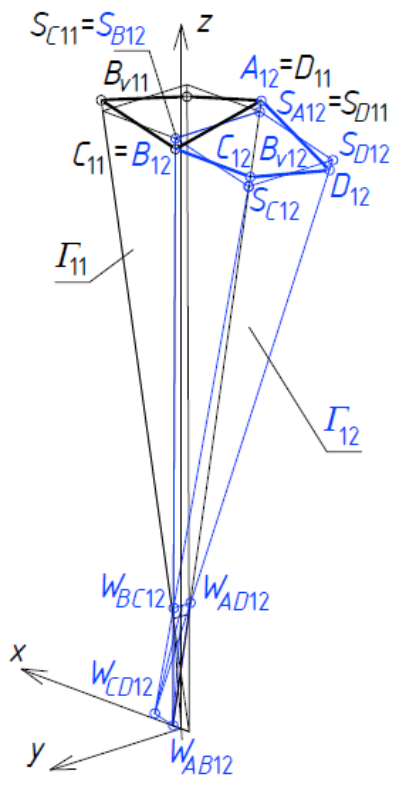

(b)

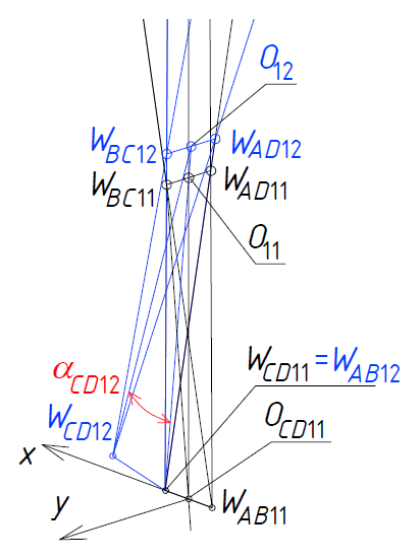

(c)

Figure 27. Creation of two initial reference tetrahedrons: (a) $\Gamma_{11} ;(\mathbf{b}) \Gamma_{12}$; and (c) a detail of $\Gamma_{12}$.

The $W_{B C 11} W_{A D 11}$ 's length results from the adopted values of the angle $\alpha_{11}$ and the height $O W_{B C 11}$ of the triangle $<W_{A B 11}, W_{C D 11}, W_{B C 11}>$ as well as the proportion.

$$
\mathrm{d}_{O W 11}=O \mathrm{~W}_{A D 11} / O W_{B C 11}
$$

The positions of points $S_{A 11}, S_{V 11}, S_{C 11}$, and $S_{D 11}$ are defined with the same constant d $\mathrm{d}_{S 11}$ listed in Table 1 as follows

$$
\begin{gathered}
\mathrm{d}_{S A 11}=m\left(W_{A B 11} S_{A 11}\right) / m\left(W_{A B 11} W_{A D 11}\right) \\
\mathrm{d}_{S B 11}=m\left(W_{A B 11} S_{B 11}\right) / m\left(W_{A B 11} W_{B C 11}\right) \\
\mathrm{d}_{S C 11}=m\left(W_{C D 11} S_{C 11}\right) / m\left(W_{C D 11} W_{B C 11}\right) \\
\mathrm{d}_{S D 11}=m\left(W_{C D 11} S_{D 11}\right) / m\left(W_{C D 11} W_{A D 11}\right) \\
\mathrm{d}_{S B 11}=\mathrm{d}_{S A 11}=\mathrm{d}_{S C 11}=\mathrm{d}_{S D 11}=\mathrm{d}_{S 11}
\end{gathered}
$$

where $W_{A B 11} \vec{W}_{A D 11}$ is the vector starting with $W_{A B 11}$ and ending at $W_{A D 11}, \mathrm{~m}\left(W_{A B 11} \vec{W}_{A D 11}\right)$ is the measure of $W_{A B 11} \vec{W}_{A D 11}, W_{A B 11} S_{A 11}$ is the vector whose starting point is $W_{A B 11}$ and ending point is $S_{A 11}$, etc. Thus, the location of points $S_{A 11}, S_{B 11}, S_{C 11}$ and $S_{D 11}$ is defined on the basis of the adopted division coefficients of all pairs $\left\{W_{A B 11}, W_{A D 11}\right\},\left\{W_{A B 11}, W_{B C 11}\right\},\left\{W_{C D 11}, W_{B C 11}\right\}$ and $\left\{W_{C D 11}, W_{A D 11}\right\}$ of the vertices of the $\Gamma_{11}$ mesh. The subsequent four points $S_{A 11}, S_{B 11}, S_{C 11}$ and $S_{D 11}$ usually form a flat rectangle determining the reference surface $\omega_{r}$ in relation to which the shell segments of the designed multi-segment shell roof are arranged in the three-dimensional space.

The locations of the vertices $A_{11}, B_{11}, C_{11}$ and $D_{11}$ of $\Gamma_{11}$, Figure $27 \mathrm{a}$, are defined by means of the following proportions

$$
\begin{aligned}
\mathrm{d}_{A 11} & =m\left(W_{A B 11} A_{11}\right) / m\left(W_{A B 11} W_{A D 11}\right) \\
\mathrm{d}_{B 11} & =m\left(W_{A B 11} B_{11}\right) / m\left(W_{A B 11} W_{B C 11}\right) \\
\mathrm{d}_{C 11} & =m\left(W_{C D 11} C_{11}\right) / m\left(W_{C D 11} W_{B C 11}\right)
\end{aligned}
$$




$$
\mathrm{d}_{D 11}=m\left(W_{C D 11} D_{11}\right) / m\left(W_{C D 11} \vec{W}_{A D 11}\right)
$$

where $W_{A B 11} A_{11}$ is the vector having the starting point at $W_{A B 11}$ and the end point at $A_{11}$, etc. Points $A_{11}, B_{11}, C_{11}$, and $D_{11}$ determine a spatial quadrangle $B_{v 11}$ constituting the eaves of a single, smooth, shell segment $\Omega_{11}$ modeling a single shell of a complex roof structure. It was adopted a constant $\mathrm{dd}_{11}$ to calculate the values of four division coefficients $\mathrm{d}_{A 11}, \mathrm{~d}_{B 11}, \mathrm{~d}_{C 11}$, and $\mathrm{d}_{D 11}$. This constant is used with positive or negative sign depending on whether the points $A_{11}, B_{11}, C_{11}$, and $D_{11}$ lie above or below $\omega_{r}$ defined in the corresponding area by means of the quadrangle $S_{A 11} S_{B 11} S_{C 11} S_{D 11}$, Figure 27a. The ratios $\mathrm{d}_{A 11}, \mathrm{~d}_{B 11}, \mathrm{~d}_{C 11}$, and $\mathrm{d}_{D 11}$ are calculated as follows

$$
\begin{aligned}
& \mathrm{d}_{A 11}=\mathrm{d}_{S A 11}+\mathrm{dd}_{A 11} \\
& \mathrm{~d}_{B 11}=\mathrm{d}_{S B 11}+\mathrm{dd}_{B 11} \\
& \mathrm{~d}_{C 11}=\mathrm{d}_{S C 11}+\mathrm{dd}_{C 11} \\
& \mathrm{~d}_{D 11}=\mathrm{d}_{S D 11}+\mathrm{dd}_{D 11} \\
& \mathrm{dd}_{B 11}=-\mathrm{dd}_{A 11}=-\mathrm{dd}_{C 11}=\mathrm{dd}_{D 11}=\mathrm{dd}_{11}
\end{aligned}
$$

In order to construct $\Gamma_{12}$, Figure $27 \mathrm{~b}$, the values presented in Table 2 were adopted.

Table 2. The initial data defining the shapes of $\Gamma_{12}$ and $B_{v 12}$.

\begin{tabular}{cc}
\hline Division Coefficient & Value \\
\hline $\mathrm{d} \alpha_{C D 12}$ & 1.2 \\
\hline $\mathrm{d}_{O 12 W C D 12}$ & 1.0 \\
$\mathrm{~d}_{W B C 12}, \mathrm{~d}_{W A D 12}$ & 1.1 \\
$\mathrm{~d}_{S C 12} \mathrm{~d}_{S D 12}$ & 2.5 \\
$\mathrm{dd}_{12}$ & 0.1 \\
\hline
\end{tabular}

The angle $\alpha_{C D 12}$ is defined by means of the angle $\alpha_{11}$ adopted in the previous step and the following formula.

$$
\mathrm{d} \alpha_{C D 12}=\alpha_{C D 12} / \alpha_{11}
$$

In order to determine $W_{C D 12}$, it was also adopted the following relationship

$$
\mathrm{d}_{O 12 W C D 12}=m\left(O_{12} \vec{W}_{C D 12}\right) / m\left(O_{12} \vec{W}_{A B 12}\right)
$$

where $O_{12}$ is the middle of the segment $W_{A D 12} W_{B C 12}$. The aforementioned values are listed in Table 2 . Two new division coefficients $\mathrm{d}_{W B C 12}$ and $\mathrm{d}_{W A D 12}$ are adopted as follows

$$
\begin{aligned}
\mathrm{d}_{W B C 12} & =m\left(W_{C D 11} \vec{W}_{B C 12}\right) / m\left(W_{C D 11} \vec{W}_{B C 11}\right) \\
\mathrm{d}_{W A D 12} & =m\left(W_{C D 11} \vec{W}_{A D 12}\right) / m\left(W_{C D 11} \vec{W}_{A D 11}\right)
\end{aligned}
$$

where $\mathrm{m}\left(\mathrm{W}_{C D 11} \vec{W}_{B C 12}\right)$ is the measure of the vector $W_{C D 11} \vec{W}_{B C 12}$ having the starting point at $W_{C D 11}$ and the end point at $W_{B C 12}$, and $\mathrm{m}\left(\mathrm{W}_{C D 11} \vec{W}_{B C 11}\right)$ is the measure of the vector $W_{C D 11} \vec{W}_{B C 11}$, etc.

The positions of points $S_{A 12}$ and $S_{B 12}$ are similar to the positions of $S_{D 11}$ and $S_{C 11}$ determined previously for $\Gamma_{11}$. The positions of $S_{C 12}$ and $S_{D 12}$ result from the following proportions

$$
\mathrm{d}_{S C 12}=\mathrm{d}_{S D 12}=\mathrm{d}_{S 11}
$$


The assumed values of these proportions are given in Table 2. The positions of points $A_{12}=D_{11}$, $B_{12}=C_{11}$ are calculated previously for $\Gamma_{11}$. The positions of points $C_{12}$ and $D_{12}$ can determined by means of the following proportions

$$
\begin{gathered}
\mathrm{d}_{C 12}=\mathrm{d}_{S C 12}+\mathrm{dd}_{C 12} \\
\mathrm{~d}_{D 12}=\mathrm{d}_{S D 12}+\mathrm{dd}_{D 12} \\
\mathrm{dd}_{C 12}=-\mathrm{dd}_{D 12}=\mathrm{dd}_{12}=\mathrm{dd}_{11}
\end{gathered}
$$

where the considered values are listed in Table 2.

The sought-after vertices $W_{A B 13}, W_{A D 13}, W_{C D 13}$, and $W_{B C 13}$ of $\Gamma_{13}$ constituting one mesh of $\Gamma_{1}$, Figure 28a, the points $S_{A 13}, S_{B 13}, S_{C 13}$, and $S_{D 13}$ of $\omega_{r}$ and the vertices $A_{13}, B_{13}, C_{13}$, and $D_{13}$ of the closed eaves quadrangle $B_{v 13}$, Figure $28 \mathrm{~b}$, can be defined analogously as for $\Gamma_{12}$ and $B_{v 12}$ using the following formula

$$
\begin{gathered}
\mathrm{d} \alpha_{C D 13}=\alpha_{C D 13} / \alpha_{11} \\
\mathrm{~d}_{O 13 W C D 13}=m\left(\vec{O}_{13} \vec{W}_{C D 13}\right) / m\left(\vec{O}_{13} \vec{W}_{A B 13}\right) \\
\mathrm{d}_{W B C 13}=m\left(\vec{W}_{C D 12} W_{B C 13}\right) / m\left(\vec{W}_{C D 12} W_{B C 12}\right) \\
\mathrm{d}_{W A D 13}=m\left(\vec{W}_{C D 12} \vec{W}_{A D 13}\right) / m\left(\vec{W}_{C D 12} W_{A D 12}\right) \\
\mathrm{d}_{S C 13}=\mathrm{d}_{S D 13}=\mathrm{d}_{S 11} \\
\mathrm{~d}_{C 13}=\mathrm{d}_{S C 13}+\mathrm{dd}_{C 13} \\
\mathrm{~d}_{D 13}=\mathrm{d}_{S D 13}+\mathrm{dd}_{D 13} \\
\mathrm{dd}_{D 13}=-\mathrm{dd}_{C 13}=\mathrm{dd}_{13}=\mathrm{dd}_{11}
\end{gathered}
$$

where $O_{13}$ is the middle point of $W_{A D 13} W_{B C 13}$.

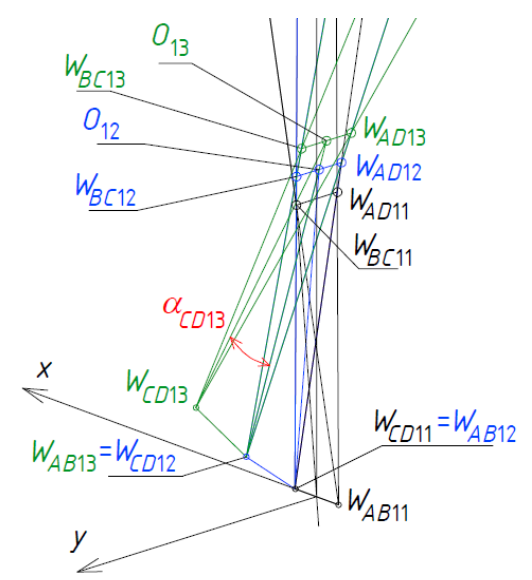

(a)

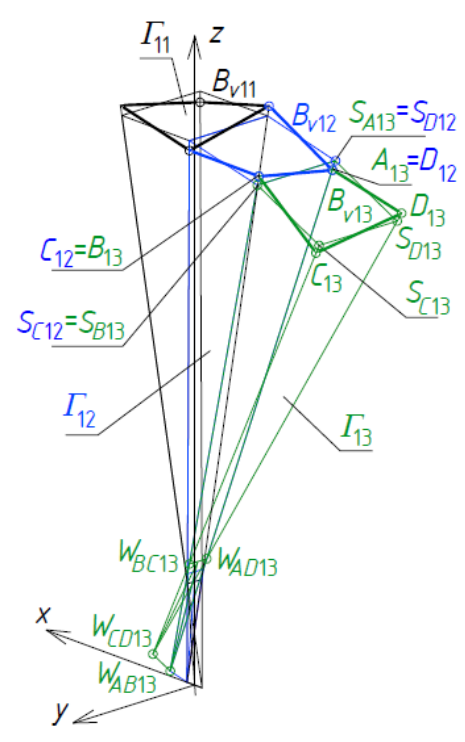

(b)

Figure 28. The step related to creation of $\Gamma_{13}$ : (a) vertices; $(\mathbf{b})$ a general shape.

The mutual position of the adjacent meshes $B_{v 12}$ and $B_{v 13}$ results from the relationships adopted for all meshes of $B_{v}$, which are as follows $S_{A 13}=S_{D 12}, S_{B 13}=S_{C 12}, A_{13}=D_{12}, B_{13}=C_{12}$, Figure 28a. The values of the adopted new proportions are given in Table 3 . 
Table 3. The initial data defining the shapes of the meshes $\Gamma_{13}$ and $B_{v 13}$ of $\Gamma$ and $B_{v}$.

\begin{tabular}{cc}
\hline Division Coefficient & Value \\
\hline $\mathrm{d} \alpha_{C D 13}$ & 1.2 \\
\hline $\mathrm{d}_{O 13 W C D 13}$ & 1.0 \\
$\mathrm{~d}_{W B C 13}, \mathrm{~d}_{W A D 13}$ & 1.1 \\
$\mathrm{~d}_{S C 13}, \mathrm{~d}_{S D 13}$ & 2.5 \\
$\mathrm{dd}_{13}$ & 0.1 \\
\hline
\end{tabular}

Compared to the investigated way for creating $\Gamma_{1 j}$ and $B_{v 1 j}$ meshes (for $j=1$ to 3 ) of the first orthogonal direction in $\Gamma$, the manner of determining the subsequent $\Gamma_{i 1}$ and $B_{v i 1}$ meshes (for $i=1$ to 3 ) of the second orthogonal direction in $\Gamma$, Figure 29a, needs a slight modification.

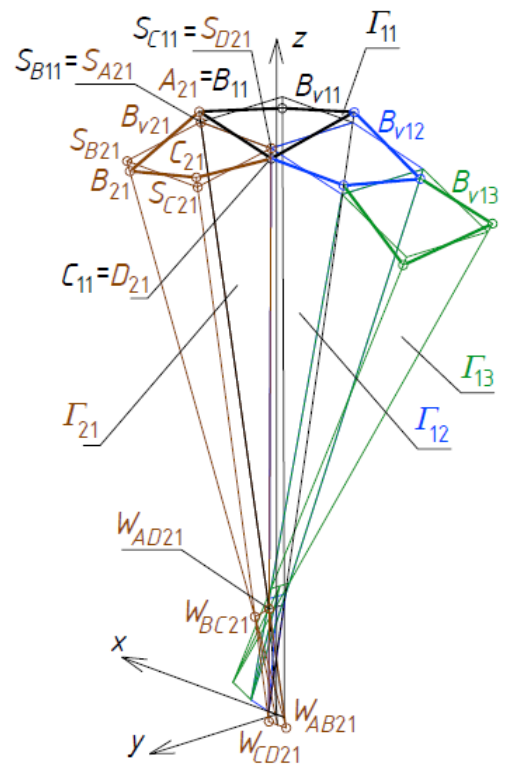

(a)

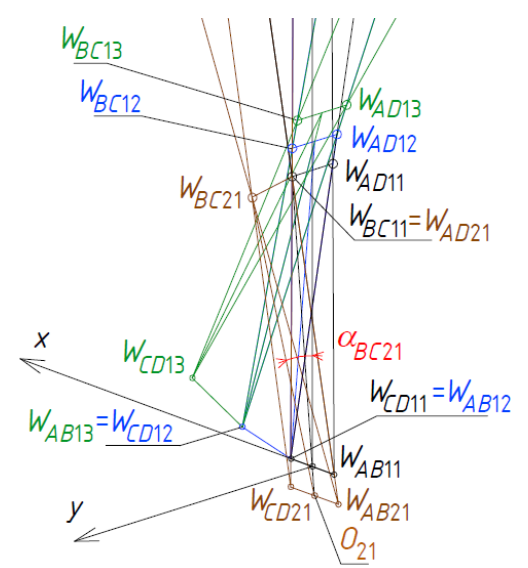

(b)

Figure 29. The step related to creation of $\Gamma_{21}$ : (a) a general shape; (b) vertices.

The angle $\alpha_{B C 21}$ is a function of the angle $\alpha_{11}$, defined with the help of the following formula

$$
\mathrm{d} \alpha_{B C 21}=\alpha_{B C 21} / \alpha_{11}
$$

The following relations were adopted

$$
\begin{gathered}
\mathrm{d}_{O 21 W B C 21}=m\left(O_{21} \vec{W}_{B C 21}\right) / m\left(O_{21} \vec{W}_{B C 11}\right) \\
\mathrm{d}_{W C D 21}=m\left(\vec{W}_{B C 11} \vec{W}_{C D 21}\right) / m\left(W_{B C 11} \vec{W}_{C D 11}\right) \\
\mathrm{d}_{W A B 21}=m\left(\vec{W}_{A D 11} \vec{W}_{C D 21}\right) / m\left(W_{A D 11} W_{C D 11}\right)
\end{gathered}
$$

where $O_{21}$ is the middle point of the edge $W_{C D 21} W_{A B 21}$, Figure 29b. The positions of points $S_{A 21}$ and $S_{D 21}$ are similar to the positions of points $S_{B 11}$ and $S_{C 11}$ obtained previously for $\Gamma_{11}$. The positions of points $S_{B 21}$ and $S_{C 21}$, Figure 29a, result from adopting of the following proportions

$$
\begin{gathered}
\mathrm{d}_{S B 21}=\mathrm{d}_{S C 21}=\mathrm{d}_{S 11} \\
\mathrm{~d}_{S B 21}=m\left(\underset{W_{A B 21}}{\vec{S} S_{B 21}}\right) / m\left(W_{A B 211} \overrightarrow{W_{B C 21}}\right)
\end{gathered}
$$




$$
\mathrm{d}_{S C 21}=m\left(W_{C D 21} S_{C 21}\right) / m\left(W_{C D 21} \vec{W}_{B C 21}\right)
$$

whose values are given in Table 4 . The positions of points $A_{21}=B_{11}$ and $D_{21}=C_{11}$. The positions of the points $B_{21}$ and $C_{21}$ result from adopting of the following proportions

$$
\begin{gathered}
\mathrm{d}_{B 21}=\mathrm{d}_{S B 21}+\mathrm{dd}_{B 21} \\
\mathrm{~d}_{C 21}=\mathrm{d}_{S C 21}+\mathrm{dd}_{C 21} \\
\mathrm{dd}_{C 21}=-\mathrm{dd}_{B 21}=\mathrm{dd}_{21}=\mathrm{dd}_{11}
\end{gathered}
$$

Table 4. The initial data defining the meshes $\Gamma_{21}$ and $B_{v 21}$.

\begin{tabular}{cc}
\hline Division Coefficient & Value \\
\hline $\mathrm{d} \alpha_{C D 21}$ & 1.2 \\
\hline $\mathrm{d}_{O 21 W B C 21}$ & 1.0 \\
$\mathrm{~d}_{W C D 21}, \mathrm{~d}_{W A B 21}$ & 1.1 \\
$\mathrm{~d}_{S B 21}, \mathrm{~d}_{S C 21}$ & 2.5 \\
$\mathrm{dd}_{21}$ & 0.1 \\
\hline
\end{tabular}

To create $\Gamma_{21}$ and $B_{v 21}$, Figure 29a, the values listed in Table 4 are employed.

All vertices $W_{A B 31}, W_{A D 31}, W_{C D 31}$ and $W_{B C 31}$ of $\Gamma_{31}$, four points $S_{A 31}, S_{B 31}, S_{C 31}$, and $S_{D 31}$ of $\omega_{r}$ and all vertices $A_{31}, B_{31}, C_{31}$, and $D_{31}$ of $B_{v 31}$ are determined like for $\Gamma_{21}$, Figure 30a,b. For this purpose, the following proportions are defined

$$
\begin{gathered}
\mathrm{d} \alpha_{\mathrm{BC} 31}=\alpha_{\mathrm{BC} 31} / \alpha_{\mathrm{BC} 21} \\
\mathrm{~d}_{O 31 W B C 31}=m\left(\vec{O}_{31} \vec{W}_{B C 31}\right) / m\left(\mathrm{O}_{31} \vec{W}_{B C 21}\right) \\
\mathrm{d}_{W C D 31}=m\left(\vec{W}_{B C 21} \vec{W}_{C D 31}\right) / m\left(\vec{W}_{B C 21} \vec{W}_{C D 21}\right) \\
\mathrm{d}_{W A B 31}=m\left(\vec{W}_{A D 21} W_{C D 31}\right) / m\left(\vec{W}_{A D 21} \vec{W}_{C D 21}\right) \\
\mathrm{d}_{S B 31}=\mathrm{d}_{S C 31}=\mathrm{d}_{S 11} \\
\mathrm{~d}_{S B 31}=m\left(\overrightarrow{W_{A B 31} S_{B 31}}\right) / m\left(W_{A B 231} W_{B C 31}\right) \\
\mathrm{d}_{S C 31}=m\left(\overrightarrow{W_{C D 31} S_{C 31}}\right) / m\left(W_{C D 31} W_{B C 31}\right) \\
\mathrm{d}_{B 31}=\mathrm{d}_{S B 31}+\mathrm{dd}_{B 31} \\
\mathrm{~d}_{C 31}=\mathrm{d}_{S C 31}+\mathrm{dd}_{C 31} \\
\mathrm{dd}_{B 31}=-\mathrm{dd}_{C 31}=\mathrm{dd}_{31}=\mathrm{dd}_{21}
\end{gathered}
$$

where $O_{31}$ is the middle point of $W_{C D 31} W_{A B 31}$. The locations of points $S_{A 31}=S_{B 21}, S_{D 31}=S_{C 21}, A_{31}=$ $B_{21}$, and $D_{31}=C_{21}$. All values of the proportions defined above are shown in Table 5 .

Table 5. The initial data defining the meshes $\Gamma_{31}$ and $B_{v 31}$.

\begin{tabular}{cc}
\hline Division Coefficient & Value \\
\hline $\mathrm{d} \alpha_{C D 31}$ & 1.0 \\
\hline $\mathrm{d}_{O 31 W B C 31}$ & 1.0 \\
$\mathrm{~d}_{W C D 31}, \mathrm{~d}_{W A B 31}$ & 1.1 \\
$\mathrm{~d}_{S B 31}, \mathrm{~d}_{S C 31}$ & 2.5 \\
$\mathrm{dd}_{31}$ & 0.1 \\
\hline
\end{tabular}




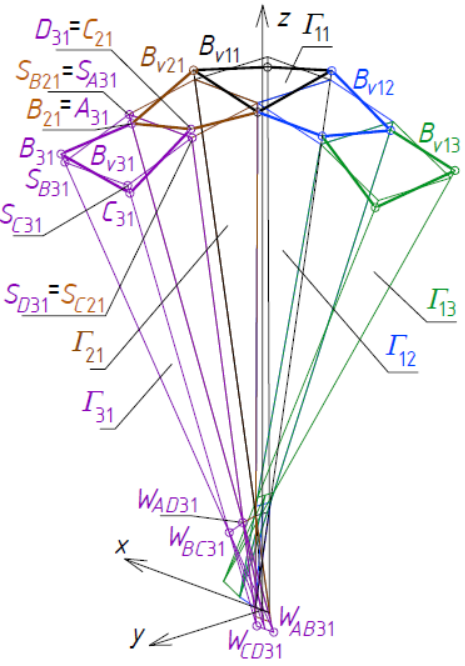

(a)

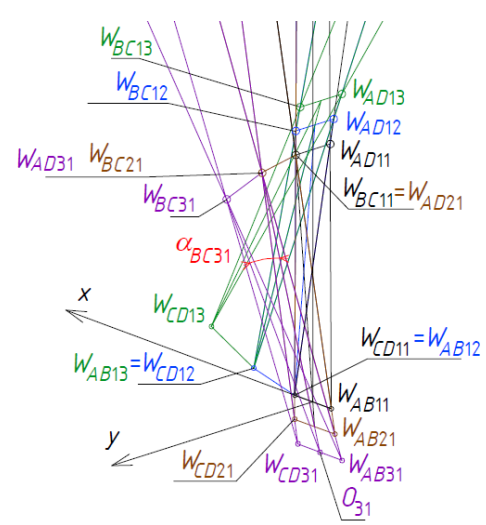

(b)

Figure 30. The step related to the creation of the reference tetrahedron $\Gamma_{31}$ : (a) a general shape; (b) vertices.

To create the tetrahedron $\Gamma_{22}$ and the quadrangle $B_{v 22}$, Figure $31 \mathrm{a}$, the values listed in Table 6 are adopted. To determine the position of $W_{C D 22}$ on the straight line $c_{22}$, Figure $31 \mathrm{a}$, c, a coefficient $\mathrm{d}_{W C D 22}$ defining the division of the edge $W_{B C 12} W_{C D 12}$ by the point $W_{C D 22}$ is defined as follows

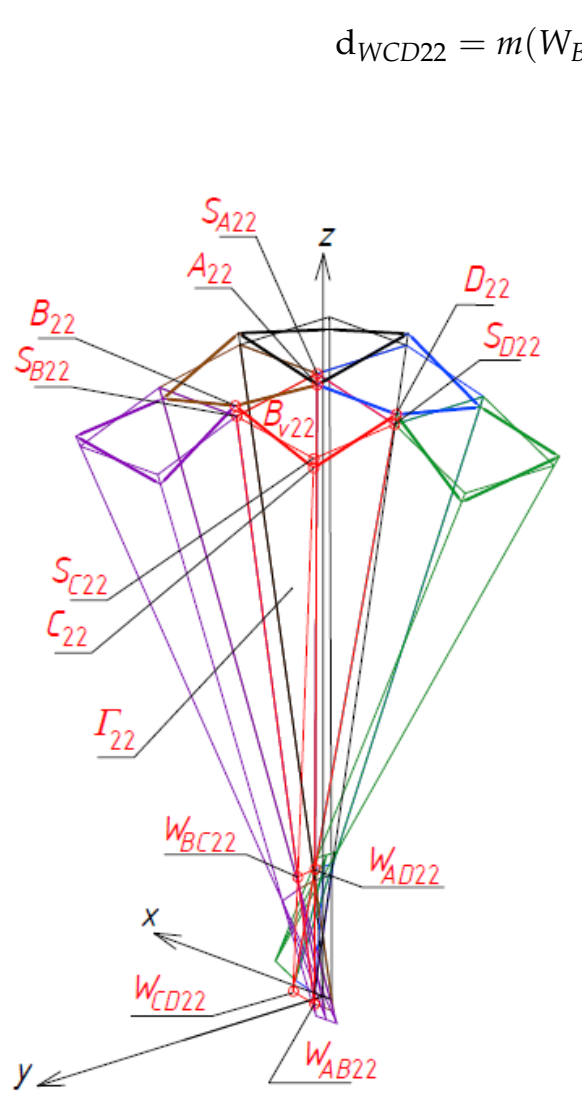

(a)

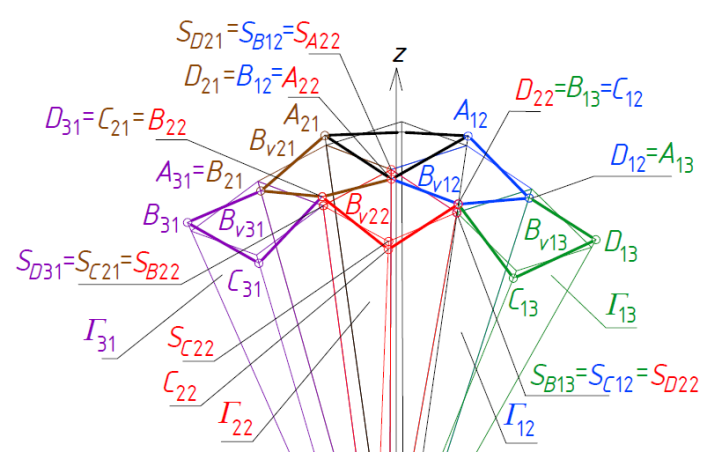

(b)

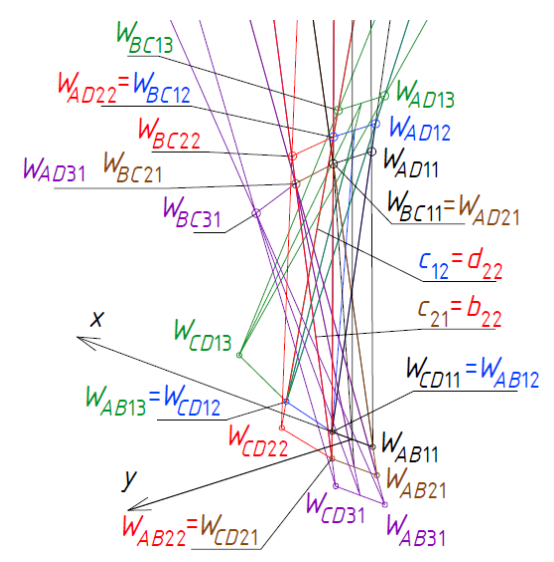

(c)

Figure 31. Determination of $\Gamma_{22}$ : (a) a general shape; (b) vertices of $B_{v 22} ;(\mathbf{c})$ vertices of $\Gamma_{22}$. 
Table 6. The initial data defining the shapes of the meshes $\Gamma_{22}$ and $B_{v 22}$.

\begin{tabular}{cc}
\hline Division Coefficient & Value \\
\hline $\mathrm{d}_{W C D 22}$ & 1.0 \\
\hline $\mathrm{d}_{W B C 22}$ & 1.0 \\
$\mathrm{~d}_{S C 22}$ & 2.5 \\
$\mathrm{dd}_{21}$ & -0.1 \\
\hline
\end{tabular}

To determine the location of $W_{B C 22}$ on $b_{22}=c_{21}$, a coefficient $\mathrm{d}_{W B C 22}$ defining the division of the edge $W_{C D 21} W_{B C 21}$ by the point $W_{B C 22}$ is assumed, Figure $31 c$, so that

$$
\mathrm{d}_{W B C 22}=m\left(W_{C D 12} \vec{W}_{B C 22}\right) / m\left(W_{C D 21} \vec{W}_{B C 21}\right)
$$

In addition, $W_{A B 22}=W_{C D 21}, W_{A D 22}=W_{B C 12}$. Similarly, the values of two coefficients $\mathrm{d}_{S C 22}$ and $\mathrm{dd}_{C 22}$ are adopted. The first value defines a division ratio of the edge $W_{C D 22} W_{B C 22}$ by $S_{C 22}$, Figure $31 c$,

$$
\mathrm{d}_{S C 22}=m\left(W_{C D 22} S_{C 22}\right) / m\left(W_{C D 22} \vec{W}_{B C 22}\right)
$$

The second proportion together with the first one enables one to define a division ratio of the edge $W_{C D 22} W_{B C 22}$ by $C_{22}$

$$
\operatorname{dd}_{S C 22}=m\left(W_{C D 22} C_{22}\right) / m\left(W_{C D 22} W_{B C 22}\right)
$$

where

$$
\mathrm{d}_{\mathrm{C} 22}=\mathrm{d}_{S C 22}+\mathrm{dd}_{S C 22}
$$

Analogous proportions as for $\Gamma_{22}$ positioned diagonally towards $\Gamma_{11}$ are defined for: (1) $\Gamma_{23}$ and $\Gamma_{32}$, located diagonally in relation to $\Gamma_{12}$ and $\Gamma_{22}$. (2) $\Gamma_{33}$ located diagonally towards $\Gamma_{22}$. Values of these proportions are listed in Table 7. A sum of all $\Gamma_{i j}$ (for $i=1-3$ ) achieved so far is a subnet $\Gamma_{1}$ constituting about one quarter of $\Gamma$. It is contained in the dihedral angle limited by the planes $(x, z)$ and $(y, z)$ containing the positive $y$-axis and negative $x$-axis, Figure 32 .

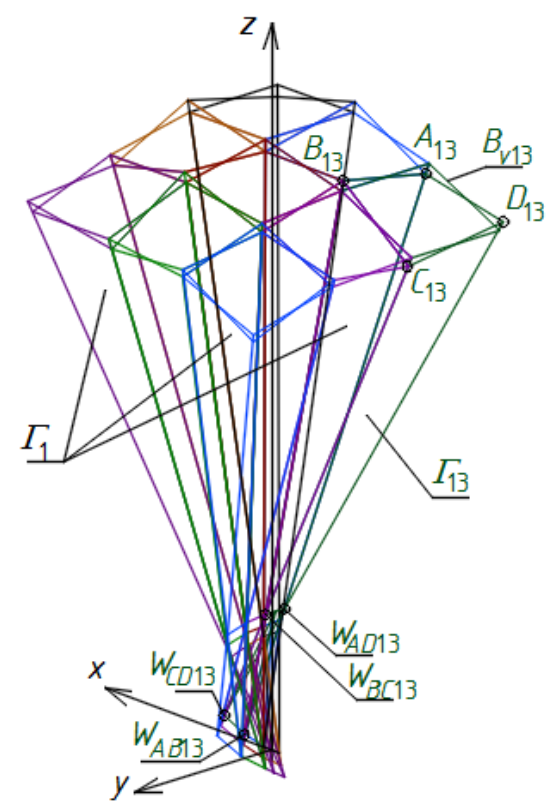

Figure 32. A quarter $\Gamma_{1}$ of the entire reference network $\Gamma$. 
Table 7. The initial data defining the meshes $\Gamma_{23}, \Gamma_{32}, \Gamma_{33}$ and $B_{v 23}, B_{v 32}, B_{v 33}$.

\begin{tabular}{cc}
\hline Division Coefficient & Value \\
\hline $\mathrm{d}_{W C D 23}$ & 1.0 \\
\hline $\mathrm{d}_{W B C 23}$ & 1.0 \\
$\mathrm{~d}_{S C 23}$ & 2.5 \\
$\mathrm{~d}_{C 23}$ & 0.1 \\
$\mathrm{~d}_{W C D 32}$ & 1.0 \\
$\mathrm{~d}_{W B C 32}$ & 1.0 \\
$\mathrm{~d}_{S C 32}$ & 2.5 \\
$\mathrm{dd}_{C 32}$ & 0.1 \\
$\mathrm{~d}_{W C D 33}$ & 1.0 \\
$\mathrm{~d}_{W B C 233}$ & 1.0 \\
$\mathrm{~d}_{S C 233}$ & 2.5 \\
$\mathrm{dd}_{C 33}$ & -0.1 \\
\hline
\end{tabular}

The reference tetrahedrons $\Gamma_{1 j}$ and $\Gamma_{i 1}$ for (for $i, j=1$ to 3 ) of the subnet $\Gamma_{1}$ were arranged in two orthogonal directions along the principal planes $(x, z)$ and $(y, z)$ of $[x, y, z]$. However, other tetrahedrons $\Gamma_{i j}$ (for $i, j=2$ to 3) are arranged in diagonal directions towards the $\Gamma_{11}$ mesh. To construct these tetrahedrons, a relatively small number of the respective proportions is employed.

The calculated coordinates of all vertices of the subnets $\Gamma_{1}$ and $B_{v 1}$ are given in Tables A1-A3 posted in Appendix A. Table A1 applies to all vertices $W_{A B i j}, W_{C D i j}, W_{A D i j}$, and $W_{B C i j}$ of $\Gamma_{1}$ (for $i, j=1$ to 3 ). Table A2 relates to the vertices $S_{A i j}, S_{B i j}, S_{C i j}$, and $S_{D i j}$ of $\omega_{r}$. Table A3 concerns all vertices $A_{i j}, B_{i j}, C_{i j}$, and $D_{i j}$ of $B_{v 1}$.

In order to create the second pair of subnets $\Gamma_{2 L}$ and $B_{v 2 L}$ of $\Gamma$ and $B_{v}$, the $z$-axis-symmetry of all characteristic points $W_{A B i j}, W_{C D i j}, W_{A D i j}, W_{B C i j}, A_{i j}, B_{i j}, C_{i j}, D_{i j}, S_{A i j}, S_{B i j}, S_{C i j}$, and $S_{D i j}$ of the previously constructed subnets $\Gamma_{1}$ and $B_{v 1}$ is used. As a result of this transformation, the vertices $W_{A B i j L}, W_{C D i j L}$, $W_{A D i j L}$ and $W_{B C i j L}$ of $\Gamma_{2 L}$, the vertices $A_{i j L}, B_{i j L}, C_{i j L}$, and $D_{i j L}$ of $B_{v i j L}$ as well as the points $S_{A i j L}, S_{B i j L}$, $S_{C i j L}$, and $S_{D i j L}$ of $\omega_{r}$ are determined so that $S_{A i j L}, S_{B i j L}, S_{C i j L}, S_{D i j L}, A_{i j L}, B_{i j L}, C_{i j L}$, and $D_{i j L}$ belong to the dihedral angle located between the $(x, z)$-plane and $(y, z)$-plane and including the positive $x$-half-axis and the negative $y$-half-axis, Figure 33. Examples of a single reference tetrahedron $\Gamma_{13 L}$ of the subnet $\Gamma_{2 L}$ and a mesh $B_{v 13 L}$ of the subnet $B_{v 2 L}$ are shown in Figure 33.
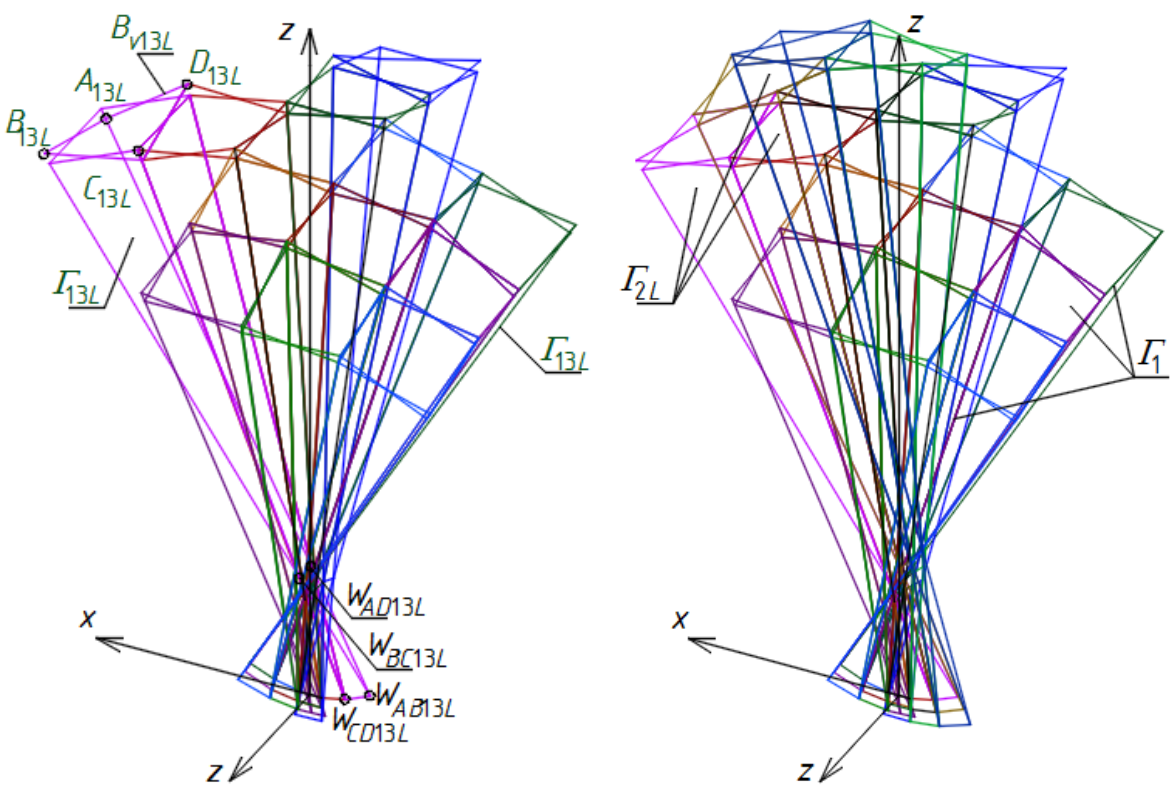

Figure 33. Two steps of the determination of the second quarter $\Gamma_{2 L}$ of the designed $z$-axis-symmetric reference network $\Gamma$. 
For the subnets $\Gamma_{2 L}$ and $B_{v 2 L}$, there are many proportions between the lengths of their side edges and axes and the measures of their angles, identical to those obtained for $\Gamma_{1}$ and $B_{v 1}$. Some selected relations resulting from the $z$-axis-symmetry of the vertices of $\Gamma_{2 L}$ and $B_{v 2 L}$ and the corresponding vertices of $\Gamma_{1}$ and $B_{v 1}$ are listed in Tables A4-A6 posted in Appendix A. Table A4 applies to the vertices $W_{A B i j L}, W_{C D i j L}, W_{A D i j L}$, and $W_{B C i j L}$ of $\Gamma_{2 L}$. Table A5 relates to the vertices $S_{A i j L}, S_{B i j L}, S_{C i j L}$, and $S_{D i j L}$ of $\omega_{r}$. Table A6 consists of the coordinates of the vertices $A_{i j L}, B_{i j L}, C_{i j L}$, and $D_{i j L}$ belonging to $B_{v 2 L}$.

To create the third pair of the subnets $\Gamma_{3 p}$ and $B_{v 3 p}$ of $\Gamma$ and $B_{v}$, a $(x, z)$-plane-symmetry, of the previously constructed nets $\Gamma_{1}$ and $B_{v 1}$ is used. Based on this symmetry, the following are transformed: (1) all vertices $W_{A B i j}, W_{C D i j}, W_{A D i j}$, and $W_{B C i j}$ of $\Gamma_{1}$, (2) all vertices $A_{i j}, B_{i j}, C_{i j}$, and $D_{i j}$ of $B_{v 1}$, (3) the points $S_{A i j}, S_{B i j}, S_{C i j}$, and $S_{D i j}$ of $\omega_{r}$. As a result of this transformation, the vertices $W_{A B i j p}, W_{C D i j p}, W_{A D i j p}$, and $W_{B C i j p}$ of $\Gamma_{3 p}$, the vertices $A_{i j p}, B_{i j p}, C_{i j p}$, and $D_{i j p}$ of $B_{v i j p}$ and the points $S_{A i j p}, S_{B i j p}, S_{C i j p}$, and $S_{D i j p}$ of $\omega_{r}$ are determined, Figure 34 . The obtained points $S_{A i j p}, S_{B i j p}, S_{C i j p}, S_{D i j p}, A_{i j p}, B_{i j p}, C_{i j p}$, and $D_{i j p}$ belong to the subspace contained between the planes $(x, z)$ and $(y, z)$, so that both the negative $x$-half-axis and the negative $y$-half-axis are included in this subspace. Examples of the reference tetrahedron $\Gamma_{23 p}$ of $\Gamma_{3 p}$ and the mesh $B_{v 23 p}$ of $B_{v 3 p}$ are shown in Figure 34 .

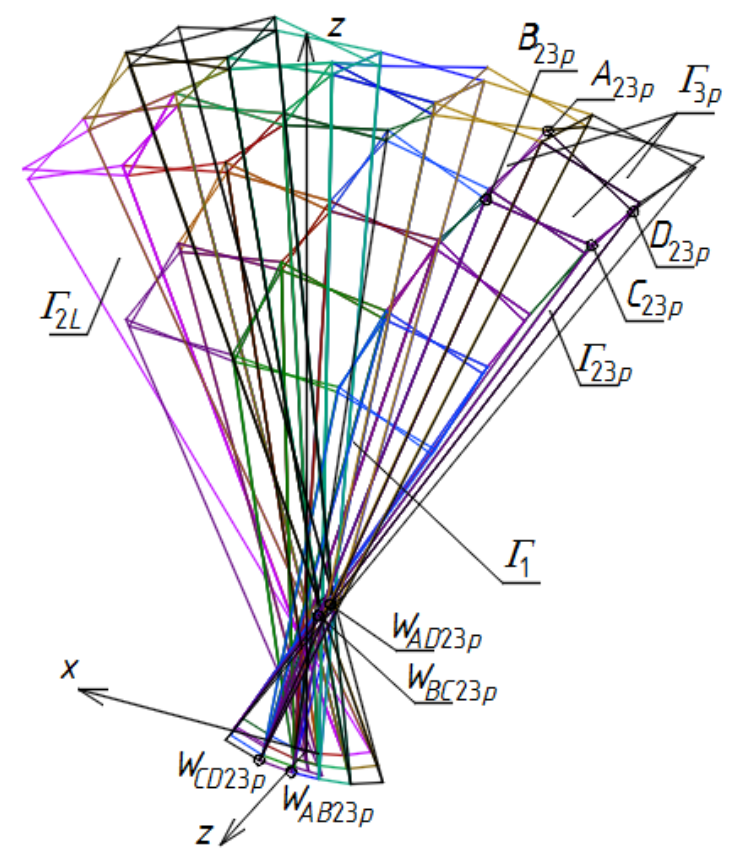

Figure 34. Three quarters $\Gamma_{1}, \Gamma_{2 L}$ and $\Gamma_{3 p}$ of the reference network $\Gamma$.

For the subnets $\Gamma_{3 p}$ and $B_{v 3 p}$, many specific proportions between their side edge and axis lengths and angle measures similar to those obtained for $\Gamma_{1}$ and $B_{v 1}$ can be found. Some relations resulting from the $(x, z)$-plane-symmetry of the vertices of $\Gamma_{3 p}$ and $B_{v 3 p}$ and the corresponding vertices of $\Gamma_{1}$ and $B_{v 1}$ created previously are listed in Tables A7-A9 posted in Appendix A. Table A7 relates to the vertices $W_{A B i j p}, W_{C D i j p}, W_{A D i j p}$, and $W_{B C i j p}$ of $\Gamma_{3 p}$. Table A8 concerns the points $S_{A i j p}, S_{B i j p}, S_{C i j p}$, and $S_{D i j p}$ of $\omega_{r}$. Table A9 applies to the vertices $A_{i j p}, B_{i j p}, C_{i j p}$, and $D_{i j p}$ of $B_{v 3 p}$.

To determine the fourth subset $\Gamma_{4 r}$ of $\Gamma$ and subnet $B_{v 4 r}$ of $B_{v}$, a $(y, z)$-plane-symmetry of $\Gamma_{1}$ and $B_{v 1}$ is used. The positions of the vertices $W_{A B i j r}, W_{C D i j r}, W_{A D i j r}$, and $W_{B C i j r}$ of $\Gamma_{4 r}$, the vertices $A_{i j r}$, $B_{i j r}, C_{i j r}$, and $D_{i j r}$ of $B_{v i j r}$ and the points $S_{A i j}, S_{B i j}, S_{C i j}$, and $S_{D i j}$ of $\omega_{r}$ are determined as a result of the transformations of (1) the vertices $W_{A B i j}, W_{C D i j}, W_{A D i j}$, and $W_{B C i j}$ of $\Gamma_{1}$, (2) the vertices $A_{i j}, B_{i j}, C_{i j}$, and $D_{i j}$ of $B_{v 1}$, (3) the points $S_{A i j}, S_{B i j}, S_{C i j}$, and $S_{D i j}$ of $\omega_{r}$, so that $S_{A i j r}, S_{B i j r}, S_{C i j r}, S_{D i j r}, A_{i j r}, B_{i j r}, C_{i j r}$, and $D_{i j r}$ belong to the dihedral angle limited by the $(x, z)$-half-plane and $(y, z)$-half-plane containing the positive $x$-half-axis and the positive $y$-half-axis, Figure 26. 
Some selected relations between the vertices of $\Gamma_{4 r}$ and $B_{v 4 r}$ and the corresponding vertices of $\Gamma_{1}$ and $B_{v 1}$, resulting from the $(y, z)$-plane-symmetry of $\Gamma$ and $B_{v}$ are given in Tables A10-A12 included in Appendix A. Table A10 relates to the $W_{A B i j r}, W_{C D i j r}, W_{A D i j r}$, and $W_{B C i j r}$ vertices of $\Gamma_{4 r}$. Table A11 concerns the points $S_{A i j r}, S_{B i j r}, S_{C i j r}$, and $S_{D i j r}$ of $\omega_{r}$. Table A12 applies to the vertices $A_{i j r}, B_{i j r}, C_{i j r}$, and $D_{i j r}$ of $B_{v 4 r}$. Finally, the sought-after nets $\Gamma$ and $B_{v}$ are created as the sums of the respective symmetric subnets $\Gamma_{1}, B_{v 1}, \Gamma_{2 L}, B_{v 2 L}, \Gamma_{3 p}, B_{v 3 p}, \Gamma_{4 r}$, and $B_{v 4 r}$ have already been constructed. These nets have to be supplemented with roof shell sectors and a plain base to obtain complete building free form model.

\section{Discussion}

The proposed method for creating parametric spatial networks enables implementation of the novel algorithms in computer programs to conveniently and intuitively search for unconventional shapes and position of elevation walls and roof shells. The benefits of the parameterization include (1) the possibility of defining and reducing a number of the independent variables entered into the method's algorithm, (2) specifying the special relations between dependent and independent variables to obtain the intuitiveness of the free form shaping, the regularity and respective curvature of the resultant complex shell roofs, (3) interesting outside roof and elevation patterns in an arrangement of many complete shell roof sectors and plane walls facets. The shape and mutual position parameterization of all reference tetrahedrons constituting the meshes of the investigated reference networks allowed developing various types of the innovative polyhedral reference networks. The activity aims at making a parametric description of these networks by means of the smallest possible number of independent variables so that such networks become regular and consistent, as well as determine diversified geometrical properties of the employed reference surfaces, including the negative, positive or zero Gaussian curvature, Figures 35 and 36.

The points $S_{A i j k}, S_{B i j k}, S_{C i j k}$, and $S_{D i j k}$ (for $i, j=1-3, k=\phi, L, p, r$, where $\phi$ is the empty set) used in the example presented in the previous section designate a regular double-curved surface $\omega_{r}$ characterized by the positive Gaussian curvature, Figures 26 and 35, because the values of the proportions $\mathrm{d}_{S A i j k}, \mathrm{~d}_{S B i j k}, \mathrm{~d}_{S C i j k}$, and $\mathrm{d}_{S D i j k}$ are bigger than 1.0. Double-curved surfaces having the negative Gaussian curvature can be obtained when the coefficients range from 0.0 to 1.0, Figure 36 . The investigated method allows one to enter certain points to determine such networks $\Gamma$ and $B_{v}$ for which the resultant reference surface is a single-curved surface having the zero Gaussian curvature. For this case, selected groups of the axes of some reference tetrahedrons $\Gamma_{i j}$ have to be contained in the same straight lines. This problem is going to be presented in further publications.

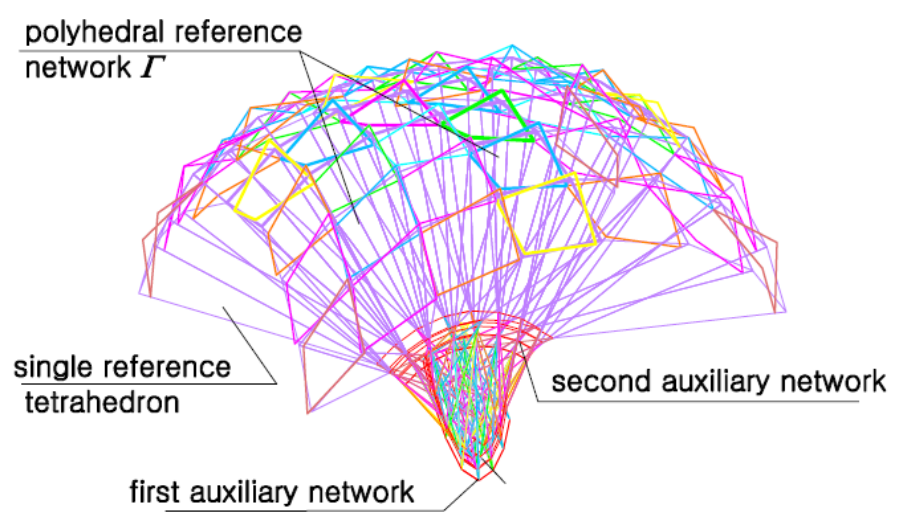

Figure 35. A sum of many spatial quadrangular meshes arranged compatible with a regular surface of the positive Gaussian curvature by means of a polyhedral reference network determined on the basis of the pair of auxiliary orthogonal networks composed of plane meshes. 


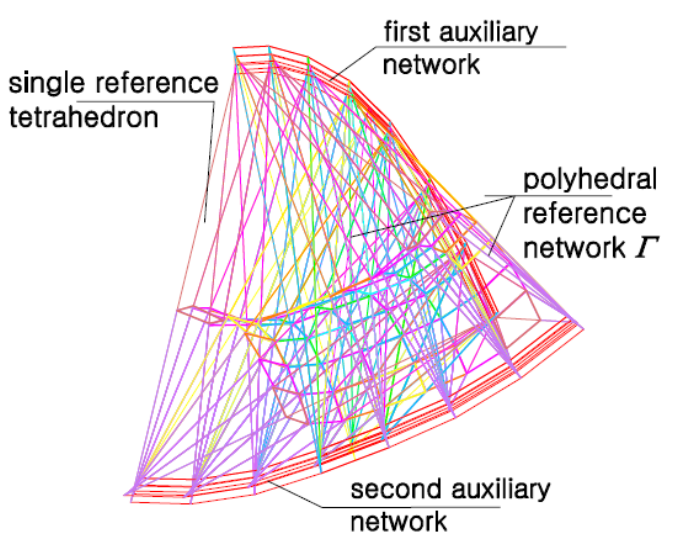

Figure 36. A sum of many spatial quadrangular meshes arranged compatible with a regular surface of the negative Gaussian curvature by means of a polyhedral reference network determined on the basis of the pair of auxiliary orthogonal networks composed of plane meshes.

In the presented example, it was shown how to create a regular, spatial, polyhedral reference network $\Gamma$ on the basis of which eaves quadrilateral network $B_{v}$ determining an unconventional shell roof structure can be built. All $\Gamma_{i j k}$ (for $i, j=1-3, k=\phi, L, p, r$ ) of $\Gamma$ are tetrahedrons whose vertices, side edges, planes and axes take specific mutual positions influencing diversified types of the created reference surfaces $\omega_{r}$ and eaves roof networks $B_{v}$. Meshes $\Gamma_{i j k}$ affect the rationality of the designed building free form structures due to the specific mutual positions of the side edges $a_{i j k}, b_{i j k}, c_{i j k}$ and $d_{i j k}$ of $\Gamma$. $B_{v i j k}$ (for $i, j=1-3, k=\phi, L, p, r$ ) of $B_{v}$ are closed spatial quadrangles whose two opposite sides can be taken as roof directrices. The parametric shapes and mutual positions of the directrices may positively affect a process of designing the attractive building structures by automatically changing the proportions $\mathrm{d}_{A i j}, \mathrm{~d}_{B i j}, \mathrm{~d}_{C i j}$, and $\mathrm{d}_{D i j}$ defining the positions of the $B_{v i j k}$ 's vertices on $a_{i j k}, b_{i j k}, c_{i j k}$ and $d_{i j k}$ in accordance with $\omega_{r}$.

The investigated method relies on special setting all subsequent reference tetrahedrons together, so that each pair of two adjacent tetrahedrons has one plane in common. Many reference tetrahedrons can be set together to obtain an edge roof shell structures having regular edge patterns on its surfaces and attractive patterns of folded plane areas on its elevations. The analyzed specific properties of the innovative spatial reference networks should lead to a creative intuitive computational shaping of attractive, rational complex-building free forms of medium and large span and novel structural systems intended for these forms.

The network $B_{v}$ introduced in Section 6, Figure 26, and the network shown in Figure 36 are characterized by the fact that each pair of their adjacent quadrilateral meshes $B_{v i j k}$ and $B_{v m n s}$ arranged orthogonally in relation to the mesh $B_{v 11}$ (for $i=1$ or $j=1$ and $m=1$ or $n=1$ and $k, s=\phi, L, p, r$ ) has one common edge, including their directrix, and two common vertices. In addition, each tetrad of adjacent quadrilaterals $B_{v i j k}$ has one common vertex. In contrast, the $B_{v}$ network shown in Figure 35 was created so that each of the two adjacent quadrangles $B_{v i j k}$ and $B_{v m n s}$ arranged in any of the orthogonal directions compatible with the principal planes $(x, z)$ and $(y, z)$ do not have a common edge, but their corresponding edges are inclined to each other and intersect in one point. In this case, each two adjacent meshes-quadrangles arranged diagonally in $B_{v}$ have only one common vertex.

The shell roof structure $\Omega$ presented in Section 6 is continuous and has many edges between smooth sectors $\Omega_{i j k}$ limited by eaves quadrangles $B_{v i j k}$ (for $i=1$ or $j=1$ and $m=1$ or $n=1$ and $k=\phi, L, p, r)$. The edges model a set of ribs between the complete transformed shells of a roof structure. In the parametric description of $\Gamma$ implemented to computer applications, it is possible to easily change the positions of all vertices $A_{i j k}, B_{i j k}, C_{i j k}, D_{i j k}$ of the meshes $B_{v i j k}$ along the side edges of $\Gamma$, relative to reference surface $\omega_{r}$ by modifying the division coefficients of some specific pairs of -the vertices of $\Gamma$. The change may cause the resultant structure $\Omega$ to become discontinuous, Figure 35 . The structure $\Omega$ can contain many empty flat areas dividing the roof shell sectors $\Omega_{i j k}$. These openings should be built 
by windows illuminating the interior of the designed building with the sunlight. This problem is also going to be analyzed in the further publications.

The author has developed some activities leading to minimize the number of independent parameters describing the geometrical properties of the presented reference networks. For this purpose, symmetrical forms of buildings must be sought. The possibility of adopting one parameter constituting only one independent variable used in defining all proportions between the selected roof and elevation elements is also developed by the author to find similar and different types of various free forms.

\section{Conclusions}

There are significant limitations in creating building free forms roofed with transformed corrugated shell sheeting concerning the complicated orthotropic geometrical and mechanical properties of thin-walled folded steel sheets. To overcome these limitations, the novel method based on the polyhedral reference networks and quadrilateral eaves nets helpful in shaping individual free forms and their specific multi-plane and multi-shell structures were carried out. As a result, the intuitive method supported by novel computer applications uses the presented relatively great possibilities of searching for diverse and innovative building structures based on the proposed shape transformations.

The main goals of combining the complete transformed shells in any roof structure include increasing the span of the roof and entire building, integrating the roof and façade forms, increasing the visual attractiveness of the entire building free form, and making it sensitive to the natural or built environments. The most common concept used in a shaping of such transformed folded shell structures is a combination of central sections of right hyperbolic paraboloids, their halves or quadrants set in various configurations, and joined along their common edges. The author developed many coherent rules for creating such complex structures covered with plane-walled folded elevations and multi-segment transformed shell roof structures. The developed algorithms allowed a radical increase in the variety of the shapes employed in design.

The presented method uses the novel vector and parametric descriptions of shaping complex building free forms characterized by the shape integration of their complex multi-shell roofs and multi-plane façades. The method's algorithm requires entering specific sets of parameters defining the general shapes of the investigated complex free forms and their individual roof and elevation elements. The parameters are either the measures of the vectors and angles of stiff motions such as translations and rotations, or the division coefficients of certain characteristic points, of the proposed novel networks by their other characteristic points.

Many proportions between geometric properties of all roof and elevations elements can be defined using functions based on the measures of the investigated types of stiff motions to achieve diversified attractive patterns on multi-plane folded elevations and multi-shell roof structures. More comprehensive studies seem to be targeted at an assignment of (a) the possible types of the independent and dependent variables, (b) the specific proportions between the dependent parameters to obtain such specific groups of the architectural free forms that are characterized by similar or different properties, (c) the search for some ranges of the values of the selected independent parameters defining attractive building free forms.

Achieving optimal, rational and attractive solutions appearing as the result of the process of shaping building free forms roofed with transformed corrugated shells and their complete elements require using regularity and symmetry of (a) single reference tetrahedrons and entire reference polyhedral networks, (b) plane walls and entire elevations of the designed complex building free forms, (c) some strips of the transformed folded sheets and entire complete transformed roofs shells and their structures, (d) structural systems intended for these forms. The research initiated and developed by the author on parametric process of architectural, geometrical, and static strength shaping of the regular free form structures roofed with transformed corrugated shell structures, and their structural systems is very extensive and requires a certain number of complete steps. One of these steps associated with 
geometrical and computational shaping such forms was elaborated by the author and the obtained results are presented in this paper.

Funding: This research was funded by Rzeszow University of Technology.

Acknowledgments: I am very grateful to A. Prokopska the head of my Department and to M. Gosztyła the Dean of my Faculty for the support.

Conflicts of Interest: The authors declare no conflict of interest. The funders had no role in the design of the study; in the collection, analyses, or interpretation of data; in the writing of the manuscript, or in the decision to publish the results.

\section{Appendix A}

Table A1. The coordinates of the vertices $W_{A B i j}, W_{C D i j}, W_{A D i j}, W_{B C i j}($ for $i, j=1,2,3)$ of the polyhedral reference network $\Gamma_{1}$ shown in Figure 27.

\begin{tabular}{|c|c|c|c|}
\hline Point & $x$-Coordinate $[\mathrm{mm}]$ & $y$-Coordinate $[\mathrm{mm}]$ & $z$-Coordinate $[\mathrm{mm}]$ \\
\hline$W_{A B 11}$ & 4500.0 & -4793.6 & $54,790.7$ \\
\hline$W_{C D 11}$ & 4500.0 & 4880.7 & $55,786.9$ \\
\hline$W_{A D 11}$ & -4500.0 & 4793.6 & $54,790.7$ \\
\hline$W_{B C 11}$ & -4500.0 & -4880.7 & $55,786.9$ \\
\hline$W_{A B 12}$ & $x W_{C D 11}$ & $y W_{C D 11}$ & $z W_{C D 11}$ \\
\hline$W_{C D 12}$ & 3254.3 & 0.0 & 468.2 \\
\hline$W_{A D 12}$ & -100.0 & -958.7 & $10,958.1$ \\
\hline$W_{B C 12}$ & -100.0 & 958.7 & $10,958.1$ \\
\hline$W_{A B 13}$ & $x W_{C D 12}$ & $y W_{C D 12}$ & $z W_{C D 12}$ \\
\hline$W_{C D 13}$ & 5572.7 & 0.0 & $z S_{C 12}$ \\
\hline$W_{A D 13}$ & -435.4 & -1054.6 & $12,007.1$ \\
\hline$W_{B C 13}$ & -435.4 & 1054.6 & $12,007.1$ \\
\hline$W_{A B 21}$ & -1100.0 & -87.2 & -996.2 \\
\hline$W_{C D 21}$ & 1100.0 & -87.2 & -996.2 \\
\hline$W_{A D 21}$ & $x W_{B C 11}$ & $y W_{B C 11}$ & $z W_{B C 11}$ \\
\hline$W_{B C 21}$ & 0.0 & 2574.0 & 9677.1 \\
\hline$W_{A B 31}$ & -1210.0 & -353.3 & -2063.5 \\
\hline$W_{C D 31}$ & 1210.0 & 353.3 & -2063.5 \\
\hline$W_{A D 31}$ & $-x W_{B C 21}$ & $y W_{B C 21}$ & $z W_{B C 21}$ \\
\hline$W_{B C 31}$ & 0.0 & 4374.6 & 9074.6 \\
\hline$W_{A B 22}$ & $x W_{C D 21}$ & $y W_{C D 21}$ & $z W_{C D 21}$ \\
\hline$W_{C D 22}$ & 3589.7 & 0.0 & -580.8 \\
\hline$W_{A D 22}$ & $x W_{B C 12}$ & $y W_{B C 12}$ & $z W_{B C 12}$ \\
\hline$W_{B C 22}$ & -110.0 & 2840.1 & $10,744.4$ \\
\hline$W_{A B 23}$ & $x W_{C D 22}$ & $y W_{C D 22}$ & $z W_{C D 22}$ \\
\hline$W_{C D 23}$ & 6173.6 & -105.5 & 435.5 \\
\hline$W_{A D 23}$ & $x W_{B C 13}$ & $y W_{B C 13}$ & $z W_{B C 13}$ \\
\hline$W_{B C 23}$ & -480.0 & 3133.7 & $11,876.9$ \\
\hline$W_{A B 32}$ & $x W_{C D 31}$ & $y W_{C D 31}$ & $z W_{C D 31}$ \\
\hline$W_{C D 32}$ & 3959.7 & -389.5 & -1713.3 \\
\hline$W_{A D 32}$ & $x W_{B C 22}$ & $y W_{B C 22}$ & $z W_{B C 22}$ \\
\hline$W_{B C 32}$ & -121.0 & 4847.4 & $10,188.4$ \\
\hline$W_{A B 33}$ & $x W_{C D 32}$ & $y W_{C D 32}$ & $z W_{C D 32}$ \\
\hline$W_{C D 33}$ & 6838.9 & -429.4 & -708.6 \\
\hline$W_{A D 33}$ & $x W_{B C 23}$ & $y W_{B C 23}$ & $z W_{B C 23}$ \\
\hline$W_{B C 33}$ & -529.1 & 5371.0 & $11,378.6$ \\
\hline
\end{tabular}


Table A2. The coordinates of the points $S_{A i j}, S_{B i j}, S_{C i j}, S_{D i j}$ for $(i, j=1,2,3)$ of the reference surface $\omega_{r}$.

\begin{tabular}{|c|c|c|c|}
\hline Point & $x$-Coordinate $[\mathrm{mm}]$ & $y$-Coordinate $[\mathrm{mm}]$ & $z$-Coordinate $[\mathrm{mm}]$ \\
\hline$S_{A 11}$ & 4500.0 & -4793.6 & $54,790.7$ \\
\hline$S_{B 11}$ & 4500.0 & 4880.7 & $55,786.9$ \\
\hline$S_{C 11}$ & -4500.0 & 4793.6 & $54,790.7$ \\
\hline$S_{D 11}$ & -4500.0 & -4880.7 & $55,786.9$ \\
\hline$S_{A 12}$ & $x S_{D 11}$ & $y S_{D 11}$ & $z S_{D 11}$ \\
\hline$S_{B 12}$ & $x S_{C 11}$ & $y S_{C 11}$ & $z S_{C 11}$ \\
\hline$S_{C 12}$ & $-13,517.1$ & 4793.6 & $52,918.0$ \\
\hline$S_{D 12}$ & $-13,517.1$ & -4793.6 & $52,918.0$ \\
\hline$S_{A 13}$ & $x S_{D 12}$ & $y S_{D 12}$ & $z S_{D 12}$ \\
\hline$S_{B 13}$ & $x S_{C 12}$ & $y S_{C 12}$ & $z S_{C 12}$ \\
\hline$S_{C 13}$ & $-21,737.1$ & 4793.6 & $49,304.2$ \\
\hline$S_{D 13}$ & $-21,737.1$ & -4793.6 & $49,304.2$ \\
\hline$S_{A 21}$ & $x S_{B 11}$ & $y S_{B 11}$ & $z S_{B 11}$ \\
\hline$S_{B 21}$ & 4500.0 & $13,460.5$ & $53,340.4$ \\
\hline$S_{C 21}$ & -4500.0 & $13,460.5$ & $53,340.4$ \\
\hline$S_{D 21}$ & $x S_{C 11}$ & $y S_{C 11}$ & $z S_{C 11}$ \\
\hline$S_{A 31}$ & $x S_{B 21}$ & $y S_{B 21}$ & $z S_{B 21}$ \\
\hline$S_{B 31}$ & 4500.0 & $21,957.5$ & $50,497.3$ \\
\hline$S_{C 31}$ & -4500.0 & $21,957.5$ & $50,497.3$ \\
\hline$S_{D 31}$ & $x S_{C 21}$ & $y S_{C 21}$ & $z S_{C 21}$ \\
\hline$S_{A 22}$ & $x S_{C 11}$ & $y S_{C 11}$ & $z S_{C 11}$ \\
\hline$S_{B 22}$ & $x S_{C 21}$ & $y S_{C 21}$ & $z S_{C 21}$ \\
\hline$S_{C 22}$ & $-13,675.6$ & $13,605.3$ & $52,270.2$ \\
\hline$S_{D 22}$ & $x S_{C 12}$ & $y S_{C 12}$ & $z S_{C 12}$ \\
\hline$S_{A 23}$ & $x S_{C 12}$ & $y S_{C 12}$ & $z S_{C 12}$ \\
\hline$S_{B 23}$ & $x S_{C 22}$ & $y S_{C 22}$ & $z S_{C 22}$ \\
\hline$S_{C 23}$ & $-22,104.0$ & $13,660.9$ & $49,061.5$ \\
\hline$S_{D 23}$ & $x S_{C 13}$ & $y S_{C 13}$ & $z S_{C 13}$ \\
\hline$S_{A 32}$ & $x S_{C 21}$ & $y S_{C 21}$ & $z S_{C 21}$ \\
\hline$S_{B 32}$ & $x S_{C 31}$ & $y S_{C 31}$ & $z S_{C 31}$ \\
\hline$S_{C 32}$ & $-13,692.4$ & $22,263.8$ & $49,770.7$ \\
\hline$S_{D 32}$ & $x S_{C 22}$ & $y S_{C 22}$ & $z S_{C 22}$ \\
\hline$S_{A 33}$ & $x S_{C 22}$ & $y S_{C 22}$ & $z S_{C 22}$ \\
\hline$S_{B 33}$ & $x S_{C 32}$ & $y S_{C 32}$ & $z S_{C 32}$ \\
\hline$S_{C 33}$ & $-22,428.4$ & $22,611.2$ & $47,304.5$ \\
\hline$S_{D 33}$ & $x S_{C 23}$ & $y S_{C 23}$ & $z S_{C 23}$ \\
\hline
\end{tabular}

Table A3. The coordinates of the vertices $A_{i j}, B_{i j}, C_{i j}, D_{i j}\left(\right.$ for $i, j=1,2,3$ ) of the eaves edge net $B_{v 1}$.

\begin{tabular}{cccc}
\hline Point & $x$-Coordinate $[\mathrm{mm}]$ & $y$-Coordinate $[\mathrm{mm}]$ & $z$-Coordinate $[\mathrm{mm}]$ \\
\hline$A_{11}$ & $4,400.0$ & $-4,706.4$ & $53,794.5$ \\
$B_{11}$ & $4,600.0$ & $4,880.7$ & $55,786.9$ \\
$C_{11}$ & $-4,400.0$ & $4,706.4$ & $53,794.5$ \\
$D_{11}$ & $-4,600.0$ & $-4,880.7$ & $55,786.9$ \\
$A_{12}$ & $-4,390.0$ & $-4,697.7$ & $53,694.9$ \\
$B_{12}$ & $-4,610.0$ & $4,889.4$ & $55,886.5$ \\
$C_{12}$ & $-13,517.1$ & $4,697.9$ & $51,869.0$ \\
$D_{12}$ & $-13,852.6$ & $-4,889.4$ & $53,967.0$ \\
$A_{13}$ & $-13,148.2$ & $-4,688.1$ & $51,764.1$ \\
$B_{13}$ & $-13,886.1$ & $4,899.0$ & $54,071.9$ \\
$C_{13}$ & $-21,136.3$ & $4,688.1$ & $48,252.2$ \\
$D_{13}$ & $-22,338.0$ & $-4,899.0$ & $50,356.2$ \\
$A_{21}$ & $4,390.0$ & $4,697.7$ & $53,694.9$ \\
$B_{21}$ & $4,610.0$ & $13,726.6$ & $54,407.7$ \\
$C_{21}$ & $-4,390.0$ & $13,194.4$ & $52,273.0$ \\
$D_{21}$ & $x B_{12}$ & $y B_{12}$ & $z B_{12}$ \\
\hline
\end{tabular}


Table A3. Cont.

\begin{tabular}{cccc}
\hline Point & $x$-Coordinate $[\mathrm{mm}]$ & $y$-Coordinate $[\mathrm{mm}]$ & $z$-Coordinate $[\mathrm{mm}]$ \\
\hline$A_{31}$ & $4,379.0$ & $13,167.7$ & $52,166.3$ \\
$B_{31}$ & $4,621.0$ & $22,430.3$ & 51611.1 \\
$C_{31}$ & $-4,379.0$ & $21,484.7$ & $49,383.5$ \\
$D_{31}$ & $-4,621.0$ & $13,753.2$ & $54,514.4$ \\
$A_{22}$ & $x C_{11}$ & $y C_{11}$ & $z C_{11}$ \\
$B_{22}$ & $x D_{31}$ & $y D_{31}$ & $z D_{31}$ \\
$C_{22}$ & $-13,367.3$ & $13,360.7$ & $51,326.4$ \\
$D_{22}$ & $x B_{13}$ & $y B_{13}$ & $z B_{13}$ \\
$A_{23}$ & $x C_{12}$ & $y C_{12}$ & $z C_{12}$ \\
$B_{23}$ & $-13,983.9$ & $13,850.0$ & $53,213.9$ \\
$C_{23}$ & $-21,549.5$ & $13,391.0$ & $48,108.1$ \\
$D_{23}$ & $-22,338.0$ & $4,899.0$ & $50,356.2$ \\
$A_{32}$ & $x C_{21}$ & $y C_{21}$ \\
$B_{32}$ & $-4,621.0$ & $22,430.3$ & $51,611.1$ \\
$C_{32}$ & $-13,352.3$ & $21,827.4$ & $48,778.9$ \\
$D_{32}$ & $x B_{23}$ & $y B_{23}$ & $z B_{23}$ \\
$A_{33}$ & $x C_{22}$ & $y C_{22}$ & $z C_{22}$ \\
$B_{33}$ & $-14,032.4$ & $22,700.2$ & $50,762.6$ \\
$C_{33}$ & $-21,916.7$ & $22,208.4$ & $46,465.1$ \\
$D_{33}$ & $-22,658.4$ & $13,930.9$ & $50,015.0$ \\
\hline
\end{tabular}

Table A4. The coordinates of the vertices $W_{A B i j L}, W_{C D i j L}, W_{A D i j L}, W_{B C i j L}($ for $i, j=1,2,3$ ) of the polyhedral reference network $\Gamma_{2 L}$ shown in Figure 28.

\begin{tabular}{|c|c|c|c|}
\hline Vertex & $x$-Coordinate $[\mathrm{mm}]$ & $y$-Coordinate $[\mathrm{mm}]$ & $z$-Coordinate $[\mathrm{mm}]$ \\
\hline$W_{A B 12 L}$ & $-x W_{C D 12}$ & $-y W_{C D 12}$ & $z W_{C D 12}$ \\
\hline$W_{C D 12 L}$ & $x W_{A B 11}$ & $y W_{A B 11}$ & $z W_{A B 11}$ \\
\hline$W_{A D 12 L}$ & $-x W_{B C 12}$ & $-y W_{B C 12}$ & $z W_{B C 12}$ \\
\hline$W_{B C 12 L}$ & $-x W_{A D 12}$ & $-y W_{A D 12}$ & $z W_{A D 12}$ \\
\hline$W_{A B 13 L}$ & $-x W_{C D 13}$ & $-y W_{C D 13}$ & $z W_{C D 13}$ \\
\hline$W_{C D 13 L}$ & $x W_{A B 12 L}$ & $y W_{A B 12 L}$ & $z W_{A B 12 L}$ \\
\hline$W_{A D 13 L}$ & $-x W_{B C 13}$ & $-y W_{B C 13}$ & $z W_{B C 13}$ \\
\hline$W_{B C 13 L}$ & $-x W_{A D 13}$ & $-y W_{A D 13}$ & $z W_{A D 13}$ \\
\hline$W_{A B 21 L}$ & $-x W_{C D 21}$ & $-y W_{C D 21}$ & $z W_{C D 21}$ \\
\hline$W_{C D 21 L}$ & $-x W_{A B 21}$ & $-y W_{A B 21}$ & $z W_{A B 21}$ \\
\hline$W_{A D 21 L}$ & $-x W_{B C 21}$ & $-y W_{B C 21}$ & $z W_{B C 21}$ \\
\hline$W_{B C 21 L}$ & $-x W_{A D 21}$ & $-y W_{A D 21}$ & $\mathrm{z} W_{A D 21}$ \\
\hline$W_{A B 31 L}$ & $-x W_{C D 31}$ & $-y W_{C D 31}$ & $z W_{C D 31}$ \\
\hline$W_{C D 31 L}$ & $-x W_{A B 31}$ & $-y W_{A B 31}$ & $z W_{A B 31}$ \\
\hline$W_{A D 31 L}$ & $-x W_{B C 31}$ & $-y W_{B C 31}$ & $z W_{B C 31}$ \\
\hline$W_{B C 31 L}$ & $-x W_{A D 31}$ & $-y W_{A D 31}$ & $\mathrm{z} W_{A D 31}$ \\
\hline$W_{A B 22 L}$ & $-x W_{C D 22}$ & $-y W_{C D 22}$ & $z W_{C D 22}$ \\
\hline$W_{C D 22 L}$ & $-x W_{A B 22}$ & $-y W_{A B 22}$ & $z W_{A B 22}$ \\
\hline$W_{A D 22 L}$ & $-x W_{B C 22}$ & $-y W_{B C 22}$ & $z W_{B C 22}$ \\
\hline$W_{B C 22 L}$ & $-x W_{A D 22}$ & $-y W_{A D 22}$ & $z W_{A D 22}$ \\
\hline$W_{A B 23 L}$ & $-x W_{C D 23}$ & $-y W_{C D 23}$ & $z W_{C D 23}$ \\
\hline$W_{C D 23 L}$ & $-x W_{A B 23}$ & $-y W_{A B 23}$ & $z W_{A B 23}$ \\
\hline$W_{A D 23 L}$ & $-x W_{B C 23}$ & $-y W_{B C 23}$ & $z W_{B C 23}$ \\
\hline$W_{B C 23 L}$ & $-x W_{A D 23}$ & $-y W_{A D 23}$ & $z W_{A D 23}$ \\
\hline$W_{A B 32 L}$ & $-x W_{C D 32}$ & $-y W_{C D 32}$ & $z W_{C D 32}$ \\
\hline$W_{C D 32 L}$ & $-x W_{A B 32}$ & $-y W_{A B 32}$ & $z W_{A B 32}$ \\
\hline$W_{A D 32 L}$ & $-x W_{B C 32}$ & $-y W_{B C 32}$ & $z W_{B C 32}$ \\
\hline$W_{B C 32 L}$ & $-x W_{A D 32}$ & $-y W_{A D 32}$ & $z W_{A D 32}$ \\
\hline$W_{A B 33 L}$ & $-x W_{C D 33}$ & $-y W_{C D 33}$ & $z W_{C D 33}$ \\
\hline$W_{C D 33 L}$ & $-x W_{A B 3}$ & $-y W_{A B 3}$ & $z W_{A B 3}$ \\
\hline$W_{A D 33 L}$ & $-x W_{B C 33}$ & $-y W_{B C 33}$ & $z W_{B C 33}$ \\
\hline$W_{B C 33 L}$ & $-x W_{A D 33}$ & $-y W_{A D 33}$ & $z W_{A D 33}$ \\
\hline
\end{tabular}


Table A5. The coordinates of the points $S_{A i j L}, S_{B i j L}, S_{C i j L}, S_{D i j L}($ for $i, j=1,2,3)$ of the reference surface $\omega_{r}$.

\begin{tabular}{|c|c|c|c|}
\hline Point & $x$-Coordinate $[\mathrm{mm}]$ & $y$-Coordinate $[\mathrm{mm}]$ & $z$-Coordinate $[\mathrm{mm}]$ \\
\hline$S_{A 12 L}$ & $-x S_{C 12}$ & $-y S_{C 12}$ & $z S_{C 12}$ \\
\hline$S_{B 12 L}$ & $-x S_{D 12}$ & $-y S_{D 12}$ & $z S_{D 12}$ \\
\hline$S_{C 12 L}$ & $-x S_{A 12}$ & $-y S_{A 12}$ & $z S_{A 12}$ \\
\hline$S_{D 12 L}$ & $-x S_{B 12}$ & $-y S_{B 12}$ & $z S_{B 12}$ \\
\hline$S_{A 13 L}$ & $-x S_{C 12}$ & $-y S_{C 13}$ & $z S_{C 13}$ \\
\hline$S_{B 13 L}$ & $-x S_{D 13}$ & $-y S_{D 13}$ & $z S_{D 13}$ \\
\hline$S_{C 13 L}$ & $-x S_{A 13}$ & $-y S_{A 13}$ & $z S_{A 13}$ \\
\hline$S_{D 13 L}$ & $-x S_{B 13}$ & $-y S_{B 13}$ & $z S_{B 13}$ \\
\hline$S_{A 21 L}$ & $-x S_{C 21}$ & $-y S_{C 21}$ & $z S_{C 21}$ \\
\hline$S_{B 21 L}$ & $-x S_{D 21}$ & $-y S_{D 21}$ & $z S_{D 21}$ \\
\hline$S_{C 21 L}$ & $-x S_{A 21}$ & $-y S_{A 21}$ & $z S_{A 21}$ \\
\hline$S_{D 21 L}$ & $-x S_{B 21}$ & $-y S_{B 21}$ & $z S_{B 21}$ \\
\hline$S_{A 31 L}$ & $-x S_{C 31}$ & $-y S_{C 31}$ & $z S_{C 31}$ \\
\hline$S_{B 31 L}$ & $-x S_{D 31}$ & $-y S_{D 31}$ & $z S_{D 31}$ \\
\hline$S_{C 31 L}$ & $-x S_{A 31}$ & $-y S_{A 31}$ & $z S_{A 31}$ \\
\hline$S_{D 31 L}$ & $-x S_{B 31}$ & $-y S_{B 31}$ & $z S_{B 31}$ \\
\hline$S_{A 22 L}$ & $-x S_{C 22}$ & $-y S_{C 22}$ & $z S_{C 22}$ \\
\hline$S_{B 22 L}$ & $-x S_{D 22}$ & $-y S_{D 22}$ & $z S_{D 22}$ \\
\hline$S_{C 22 L}$ & $-x S_{A 22}$ & $-y S_{A 22}$ & $z S_{A 22}$ \\
\hline$S_{D 22 L}$ & $-x S_{B 22}$ & $-y S_{B 22}$ & $z S_{B 22}$ \\
\hline$S_{A 23 L}$ & $-x S_{C 23}$ & $-y S_{C 23}$ & $z S_{C 23}$ \\
\hline$S_{B 23 L}$ & $-x S_{D 23}$ & $-y S_{D 23}$ & $z S_{D 23}$ \\
\hline$S_{C 23 L}$ & $-x S_{A 23}$ & $-y S_{A 23}$ & $z S_{A 23}$ \\
\hline$S_{D 23 L}$ & $-x S_{B 23}$ & $-y S_{B 23}$ & $z S_{B 23}$ \\
\hline$S_{A 32 L}$ & $-x S_{C 32}$ & $-y S_{C 32}$ & $z S_{C 32}$ \\
\hline$S_{B 32 L}$ & $-x S_{D 32}$ & $-y S_{D 32}$ & $z S_{D 32}$ \\
\hline$S_{C 32 L}$ & $-x S_{A 32}$ & $-y S_{A 32}$ & $z S_{A 32}$ \\
\hline$S_{D 32 L}$ & $-x S_{B 32}$ & $-y S_{B 32}$ & $z S_{B 32}$ \\
\hline$S_{A 33 L}$ & $-x S_{C 33}$ & $-y S_{C 33}$ & $z S_{C 33}$ \\
\hline$S_{B 33 L}$ & $-x S_{D 33}$ & $-y S_{D 33}$ & $z S_{D 33}$ \\
\hline$S_{C 33 L}$ & $-x S_{A 33}$ & $-y S_{A 33}$ & $z S_{A 33}$ \\
\hline$S_{D 33 L}$ & $-x S_{B 33}$ & $-y S_{B 33}$ & $z S_{B 33}$ \\
\hline
\end{tabular}

Table A6. The coordinates of the vertices $A_{i j L}, B_{i j L}, C_{i j L}, D_{i j L}$ (for $i, j=1,2,3$ ) of the eaves edge net $B_{v 2 L}$.

\begin{tabular}{cccc}
\hline Point & $x$-Coordinate $[\mathrm{mm}]$ & $y$-Coordinate $[\mathrm{mm}]$ & $z$-Coordinate $[\mathrm{mm}]$ \\
\hline$A_{12 L}$ & $-x C_{12}$ & $-y C_{12}$ & $z C_{12}$ \\
$B_{12 L}$ & $-x D_{12}$ & $-y D_{12}$ & $z D_{12}$ \\
$C_{12 L}$ & $-x A_{12}$ & $-y A_{12}$ & $z A_{12}$ \\
$D_{12 L}$ & $-x B_{12}$ & $-y B_{12}$ & $z B_{12}$ \\
$A_{13 L}$ & $-x C_{13}$ & $-y C_{13}$ & $z C_{13}$ \\
$B_{13 L}$ & $-x D_{13}$ & $-y D_{13}$ & $z D_{13}$ \\
$C_{13 L}$ & $-x A_{13}$ & $-y A_{13}$ & $z A_{13}$ \\
$D_{13 L}$ & $-x B_{13}$ & $-y B_{13}$ & $z B_{13}$ \\
$A_{21 L}$ & $-x C_{21}$ & $-y C_{21}$ & $z C_{21}$ \\
$B_{21 L}$ & $-x D_{21}$ & $-y D_{21}$ & $z D_{21}$ \\
$C_{21 L}$ & $-x A_{21}$ & $-y A_{21}$ & $z A_{21}$ \\
$D_{21 L}$ & $-x B_{21}$ & $-y B_{21}$ & $z B_{21}$ \\
$A_{31 L}$ & $-x C_{31}$ & $-y C_{31}$ & $z C_{31}$ \\
$B_{31 L}$ & $-x D_{31}$ & $-y D_{31}$ & $z D_{31}$ \\
$C_{31 L}$ & $-x A_{31}$ & $-y A_{31}$ & $z A_{31}$ \\
$D_{31 L}$ & $-x B_{31}$ & $-y B_{31}$ & $z B_{31}$ \\
$A_{22 L}$ & $-x C_{22}$ & $-y C_{22}$ & $z C_{22}$ \\
$B_{22 L}$ & $-x D_{22}$ & $-y D_{22}$ & $z D_{22}$ \\
$C_{22 L}$ & $-x A_{22}$ & $-y A_{22}$ & $z A_{22}$ \\
$D_{22 L}$ & $-x B_{22}$ & $-y B_{22}$ & $z B_{22}$ \\
\hline
\end{tabular}


Table A6. Cont.

\begin{tabular}{cccc}
\hline Point & $x$-Coordinate $[\mathrm{mm}]$ & $y$-Coordinate $[\mathrm{mm}]$ & $z$-Coordinate $[\mathrm{mm}]$ \\
\hline$A_{23 L}$ & $-x C_{23}$ & $y C_{23}$ & $z C_{23}$ \\
$B_{23 L}$ & $-x D_{23}$ & $-y D_{23}$ & $z D_{23}$ \\
$C_{23 L}$ & $-x A_{23}$ & $-y A_{23}$ & $z A_{23}$ \\
$D_{23 L}$ & $-x B_{23}$ & $-y B_{23}$ & $z B_{23}$ \\
$A_{32 L}$ & $-x C_{32}$ & $-y C_{32}$ & $z C_{32}$ \\
$B_{32 L}$ & $-x D_{32}$ & $-y D_{32}$ & $z D_{32}$ \\
$C_{32 L}$ & $-x A_{32}$ & $-y A_{32}$ & $z A_{32}$ \\
$D_{32 L}$ & $-x B_{32}$ & $-y B_{32}$ & $z B_{32}$ \\
$A_{33 L}$ & $-x C_{33}$ & $-y C_{33}$ & $z C_{33}$ \\
$B_{33 L}$ & $-x D_{33}$ & $-y D_{33}$ & $z D_{33}$ \\
$C_{33 L}$ & $-x A_{33}$ & $-y A_{33}$ & $z A_{33}$ \\
$D_{33 L}$ & $-x B_{33} 0$ & $-y B_{33}$ & $z B_{33}$ \\
\hline
\end{tabular}

Table A7. The coordinates of the vertices $W_{A B i j p}, W_{C D i j p}, W_{A D i j p}, W_{B C i j p}$ (for $i, j=1,2,3$ ) of the polyhedral reference network $\Gamma_{3 p}$ shown in Figure 29.

\begin{tabular}{cccc}
\hline Vertex & $x$-Coordinate [mm] & $y$-Coordinate [mm] & $z$-Coordinate [mm] \\
\hline$W_{A B 22 p}$ & $x W_{A B 22}$ & $-y W_{A B 22}$ & $z W_{A B 22}$ \\
$W_{C D 22 p}$ & $x W_{C D 22}$ & $-y W_{C D 22}$ & $z W_{C D 22}$ \\
$W_{A D 22 p}$ & $x W_{B C 22}$ & $-y W_{B C 22}$ & $z W_{B C 22}$ \\
$W_{B C 22 p}$ & $x W_{A D 22}$ & $-y W_{A D 22}$ & $z W_{A D 22}$ \\
$W_{A B 23 p}$ & $x W_{A B 23}$ & $-y W_{A B 23}$ & $z W_{A B 23}$ \\
$W_{C D 23 p}$ & $x W_{C D 23}$ & $-y W_{C D 23}$ & $z W_{C D 23}$ \\
$W_{A D 23 p}$ & $x W_{B C 23}$ & $-y W_{B C 23}$ & $z W_{B C 23}$ \\
$W_{B C 23 p}$ & $x W_{A D 23}$ & $-y W_{A D 23}$ & $z W_{A D 23}$ \\
$W_{A B 32 p}$ & $x W_{A B 32}$ & $-y W_{A B 32}$ & $z W_{A B 32}$ \\
$W_{C D 32 p}$ & $x W_{C D 32}$ & $-y W_{C D 32}$ & $z W_{C D 32}$ \\
$W_{A D 32 p}$ & $x W_{B C 32}$ & $-y W_{B C 32}$ & $z W_{B C 32}$ \\
$W_{B C 32 p}$ & $x W_{A D 32}$ & $-y W_{A D 32}$ & $z W_{A D 32}$ \\
$W_{A B 33 p}$ & $x W_{A B 33}$ & $-y W_{A B 33}$ & $z W_{A B 33}$ \\
$W_{C D 33 p}$ & $x W_{C D 33}$ & $-y W_{C D 33}$ & $z W_{C D 33}$ \\
$W_{A D 33 p}$ & $x W_{B C 33}$ & $-y W_{B C 33}$ & $z W_{B C 33}$ \\
$W_{B C 33 p}$ & $x W_{A D 33}$ & $-y W_{A D 33}$ & $z W_{A D 33}$ \\
\hline
\end{tabular}

Table A8. The coordinates of the points $S_{\text {Aijp }}, S_{\text {Bijp }}, S_{\text {Cijp }}, S_{\text {Dijp }}$ for $(i, j=1,2,3)$ of the reference surface $\omega_{r}$.

\begin{tabular}{cccc}
\hline Point & $x$-Coordinate [mm] & $y$-Coordinate [mm] & $z$-Coordinate [mm] \\
\hline$S_{A 22 p}$ & $x S_{B 22}$ & $-y S_{B 22}$ & $z S_{B 22}$ \\
$S_{B 22 p}$ & $x S_{A 22}$ & $-y S_{A 22}$ & $z S_{A 22}$ \\
$S_{C 22 p}$ & $x S_{D 22}$ & $-y S_{D 22}$ & $z S_{D 22}$ \\
$S_{D 22 p}$ & $x S_{C 22}$ & $-y S_{C 22}$ & $z S_{C 22}$ \\
$S_{A 23 p}$ & $x S_{B 23}$ & $-y S_{B 23}$ & $z S_{B 23}$ \\
$S_{B 23 p}$ & $x S_{A 23}$ & $-y S_{A 23}$ & $z S_{A 23}$ \\
$S_{C 23 p}$ & $x S_{D 23}$ & $-y S_{D 23}$ & $z S_{D 23}$ \\
$S_{D 23 p}$ & $x S_{C 23}$ & $-y S_{C 23}$ & $z S_{C 23}$ \\
$S_{A 32 p}$ & $x S_{B 32}$ & $-y S_{B 32}$ & $z S_{B 32}$ \\
$S_{B 32 p}$ & $x S_{A 32}$ & $-y S_{A 32}$ & $z S_{A 32}$ \\
$S_{C 32 p}$ & $x S_{D 32}$ & $-y S_{D 32}$ & $z S_{D 32}$ \\
$S_{D 32 p}$ & $x S_{C 32}$ & $-y S_{C 32}$ & $z S_{C 32}$ \\
$S_{A 33 p}$ & $x S_{B 33}$ & $-y S_{B 33}$ & $z S_{B 33}$ \\
$S_{B 33 p}$ & $x S_{A 33}$ & $-y S_{A 33}$ & $z S_{A 33}$ \\
$S_{C 33 p}$ & $x S_{D 33}$ & $-y S_{D 33}$ & $z S_{D 33}$ \\
$S_{D 33 p}$ & $x S_{C 33}$ & $-y S_{C 33}$ & $z S_{C 33}$ \\
\hline
\end{tabular}


Table A9. The coordinates of the vertices $A_{i j p}, B_{i j p}, C_{i j p}, D_{i j p}$, (for $\left.i, j=1,2,3\right)$ of the eaves edge net $B_{v 3 p}$.

\begin{tabular}{cccc}
\hline Point & $x$-Coordinate $[\mathrm{mm}]$ & $y$-Coordinate $[\mathrm{mm}]$ & $z$-Coordinate $[\mathrm{mm}]$ \\
\hline$A_{22 p}$ & $x A_{32}$ & $-y A_{32}$ & $z A_{32}$ \\
$B_{22 p}$ & $x B_{12}$ & $-y B_{12}$ & $z B_{12}$ \\
$C_{22 p}$ & $x C_{12}$ & $-y C_{12}$ & $z C_{12}$ \\
$D_{22 p}$ & $x D_{32}$ & $-y D_{32}$ & $z D_{32}$ \\
$A_{23 p}$ & $x A_{33}$ & $-y A_{33}$ & $z A_{33}$ \\
$B_{23 p}$ & $x B_{13}$ & $-y B_{13}$ & $z B_{13}$ \\
$C_{23 p}$ & $x C_{13}$ & $-y C_{13}$ & $z C_{13}$ \\
$D_{23 p}$ & $x D_{33}$ & $-y D_{33}$ & $z D_{33}$ \\
$A_{32 p}$ & $x C_{31}$ & $-y C_{31}$ & $z C_{31}$ \\
$B_{32 p}$ & $x B_{22}$ & $-y B_{22}$ & $z B_{22}$ \\
$C_{32 p}$ & $x C_{22}$ & $-y C_{22}$ & $z C_{22}$ \\
$D_{32 p}$ & $x B_{33}$ & $-y B_{33}$ & $z B_{33}$ \\
$A_{33 p}$ & $x C_{32}$ & $-y C_{32}$ & $z C_{32}$ \\
$B_{33 p}$ & $x B_{23}$ & $-y B_{23}$ & $z B_{23}$ \\
$C_{33 p}$ & $x C_{23}$ & $-y C_{23}$ & $z C_{23}$ \\
$D_{33 p}$ & $-22,940.0$ & $23,014.0$ & $48,143.90$ \\
\hline
\end{tabular}

Table A10. The coordinates of the vertices $W_{A B i j r}, W_{C D i j r}, W_{A D i j r}, W_{B C i j r}($ for $i, j=1,2,3)$ of the polyhedral reference network $\Gamma_{4 r}$ shown in Figure 21.

\begin{tabular}{cccc}
\hline Vertex & $x$-Coordinate [mm] & $y$-Coordinate [mm] & $z$-Coordinate [mm] \\
\hline$W_{A B 22 r}$ & $-x W_{C D 22}$ & $y W_{C D 22}$ & $z W_{C D 22}$ \\
$W_{C D 22 r}$ & $-x W_{A B 22}$ & $y W_{A B 22}$ & $z W_{A B 22}$ \\
$W_{A D 22 r}$ & $-x W_{A D 22}$ & $y W_{A D 22}$ & $z W_{A D 22}$ \\
$W_{B C 22 r}$ & $-x W_{B C 22}$ & $y W_{B C 22}$ & $z W_{B C 22}$ \\
$W_{A B 23 r}$ & $-x W_{C D 23}$ & $y W_{C D 23}$ & $z W_{C D 23}$ \\
$W_{C D 23 r}$ & $-x W_{A B 23}$ & $y W_{A B 23}$ & $z W_{A B 23}$ \\
$W_{A D 23 r}$ & $-x W_{A D 23}$ & $y W_{A D 23}$ & $z W_{A D 23}$ \\
$W_{B C 23 r}$ & $-x W_{B C 23}$ & $y W_{B C 23}$ & $z W_{B C 23}$ \\
$W_{A B 32 r}$ & $-x W_{C D 32}$ & $y W_{C D 32}$ & $z W_{C D 32}$ \\
$W_{C D 32 r}$ & $-x W_{A B 32}$ & $y W_{A B 32}$ & $z W_{A B 32}$ \\
$W_{A D 32 r}$ & $-x W_{A D 32}$ & $y W_{A D 32}$ & $z W_{A D 32}$ \\
$W_{B C 32 r}$ & $-x W_{B C 32}$ & $y W_{B C 32}$ & $z W_{B C 32}$ \\
$W_{A B 33 r}$ & $-x W_{C D 33}$ & $y W_{C D 33}$ & $z W_{C D 33}$ \\
$W_{C D 33 r}$ & $-x W_{A B 33}$ & $y W_{A B 33}$ & $z W_{A B 33}$ \\
$W_{A D 33 r}$ & $-x W_{A D 33}$ & $y W_{A D 33}$ & $z W_{A D 33}$ \\
$W_{B C 33 r}$ & $-x W_{B C 33}$ & $y W_{B C 33}$ & $z W_{B C 33}$ \\
\hline
\end{tabular}

Table A11. The coordinates of the points $S_{A i j r}, S_{B i j r}, S_{C i j r}, S_{D i j r}$ for $(i, j=1,2,3)$ of the reference surface $\omega_{r}$.

\begin{tabular}{cccc}
\hline Point & $x$-Coordinate $[\mathrm{mm}]$ & $y$-Coordinate [mm] & $z$-Coordinate [mm] \\
\hline$S_{A 22 r}$ & $-x S_{D 22}$ & $y S_{D 22}$ & $z S_{D 22}$ \\
$S_{B 22 r}$ & $-x S_{C 22}$ & $y S_{C 22}$ & $z S_{C 22}$ \\
$S_{C 22 r}$ & $-x S_{B 22}$ & $y S_{B 22}$ & $z S_{B 22}$ \\
$S_{D 22 r}$ & $-x S_{A 22}$ & $y S_{A 22}$ & $z S_{A 22}$ \\
$S_{A 23 r}$ & $-x S_{D 23}$ & $y S_{D 23}$ & $z S_{D 23}$ \\
$S_{B 23 r}$ & $-x S_{C 23}$ & $y S_{C 23}$ & $z S_{C 23}$ \\
$S_{C 23 r}$ & $-x S_{B 23}$ & $y S_{B 23}$ & $z S_{B 23}$ \\
$S_{D 23 r}$ & $-x S_{A 23}$ & $y S_{A 23}$ & $z S_{A 23}$ \\
$S_{A 32 r}$ & $-x S_{D 32}$ & $y S_{D 32}$ & $z S_{D 32}$ \\
$S_{B 32 r}$ & $-x S_{C 32}$ & $y S_{C 32}$ & $z S_{C 32}$ \\
$S_{C 32 r}$ & $-x S_{B 32}$ & $y S_{B 32}$ & $z S_{B 32}$ \\
$S_{D 32 r}$ & $-x S_{A 32}$ & $y S_{A 32}$ & $z S_{A 32}$ \\
$S_{A 33 r}$ & $-x S_{D 33}$ & $y S_{D 33}$ & $z S_{D 33}$ \\
$S_{B 33 r}$ & $-x S_{C 33}$ & $y S_{C 33}$ & $z S_{C 33}$ \\
$S_{C 33 r}$ & $-x S_{B 33}$ & $y S_{B 33}$ & $z S_{B 33}$ \\
$S_{D 33 r}$ & $-x S_{A 33}$ & $y S_{A 33}$ & $z S_{A 33}$ \\
\hline
\end{tabular}


Table A12. The coordinates of the vertices $A_{i j r}, B_{i j r}, C_{i j r}, D_{i j r}$ (for $i, j=1,2,3$ ) of the eaves edge net $B_{v 4 r}$.

\begin{tabular}{cccc}
\hline Point & $x$-Coordinate $[\mathrm{mm}]$ & $y$-Coordinate $[\mathbf{m m}]$ & $z$-Coordinate $[\mathrm{mm}]$ \\
\hline$A_{22 r}$ & $-x A_{23}$ & $y A_{23}$ & $z A_{23}$ \\
$B_{22 r}$ & $-x B_{23}$ & $y B_{23}$ & $z B_{23}$ \\
$C_{22 r}$ & $-x C_{21}$ & $y C_{21}$ & $z C_{21}$ \\
$D_{22 r}$ & $-x D_{21}$ & $y D_{21}$ & $z D_{21}$ \\
$A_{23 r}$ & $-x C_{13}$ & $y C_{13}$ & $z C_{13}$ \\
$B_{23 r}$ & $-x D_{33}$ & $y D_{33}$ & $z D_{33}$ \\
$C_{23 r}$ & $-x C_{22}$ & $y C_{22}$ & $z C_{22}$ \\
$D_{23 r}$ & $-x D_{22}$ & $y D_{22}$ & $z D_{22}$ \\
$A_{32 r}$ & $-x A_{33}$ & $y A_{33}$ & $z A_{33}$ \\
$B_{32 r}$ & $-x B_{33}$ & $y B_{33}$ & $z B_{33}$ \\
$C_{32 r}$ & $-x C_{31}$ & $y C_{31}$ & $z C_{31}$ \\
$D_{32 r}$ & $-x D_{31}$ & $y D_{31}$ & $z D_{31}$ \\
$A_{33 r}$ & $-x C_{23}$ & $y C_{23}$ & $z C_{23}$ \\
$B_{33 r}$ & $-x D_{33 p}$ & $-y D_{33 p}$ & $z D_{33 p}$ \\
$C_{33 r}$ & $-x C_{32}$ & $y C_{32}$ & $z C_{32}$ \\
$D_{33 r}$ & $-x D_{32}$ & $y D_{32}$ & $z D_{32}$ \\
\hline
\end{tabular}

\section{References}

1. Yu, W.-W.; LaBoube, R.A.; Chen, H. Cold Formed Steel Design; John Wiley and Sons Inc.: New York, NY, USA, 2000.

2. Abdel_Sayed, G. Critical shear loading of curved panels of corrugated sheets. Proc. ASCE J. Struct. Div. 1970, 96, 1279-1294.

3. Abramczyk, J. Shell Free Forms of Buildings Roofed with Transformed Corrugated Sheeting, Monograph; Publishing House of Rzeszow University of Technology: Rzeszów, Poland, 2017.

4. Parker, J.E. Behavior of Light Gauge Steel Hyperbolic Paraboloid Shells. Ph.D. Thesis, Cornell University, Ithaca, NY, USA, 1969.

5. Petcu, V.; Gioncu, D. Corrugated hypar structures. In Proceedings of the I International Conference on Lightweight Structures in Civil Engineering, Warsaw, Poland, 1 December 1995; pp. 637-644.

6. Yang, H. A Finite Element Formulation for Stability Analysis of Doubly Curved Thin Shell Structures. Ph.D. Thesis, Cornell University, Ithaca, NY, USA, 1969.

7. Prokopska, A.; Abramczyk, J. Parametric Creative Design of Building Free Forms Roofed with Transformed Shells Introducing Architect's and Civil Engineer's Responsible Artistic Concepts. Buildings 2019, 9, 58. [CrossRef]

8. Abramczyk, J. Shape transformations of folded sheets providing shell free forms for roofing. In Proceedings of the 11th Conference on Shell Structures: Theory and Applications, Gdańsk, Poland, 11-13 October 2017; Pietraszkiewicz, W., Witowski, W., Taylor and Francis Group, Eds.; Routledge: London, UK, 2017; Volume 4, pp. 409-412.

9. Prokopska, A.; Abramczyk, J. Shape Transformations of Plane Folded Sheets for Shell Roofing. IOP Conf. Ser. Mater. Sci. Eng. 2019, 471, 082064. [CrossRef]

10. Reichhart, A. Geometrical and Structural Shaping Building Shells Made up of Transformed Flat Folded Sheets; Hhouse of Rzeszow University of Technology: Rzeszów, Poland, 2002. (In Polish)

11. Abramczyk, J. Building Structures Roofed with Multi-Segment Corrugated Hyperbolic Paraboloid Steel Shells. Procedia Eng. 2016, 161, 1545-1550. [CrossRef]

12. Nilson, V.E. Testing a light gauge steel hyperbolic paraboloid shell. Proc. ASCE J. Struct. Div. 1962, 88, 51-66.

13. Winter, G. Strength of thin steel compression flanges. Trans. ASCE 1974, 112, 895-912.

14. Muskat, R. Buckling of Light Gage Steel Hyperbolic Paraboloid Roofs. Ph.D. Thesis, Cornell University, Ithaca, NY, USA, 1968.

15. Banavalkar, P.V. Analysis and Behavior of Light Gauge Hyperbolic Paraboloid Shells. Ph.D. Thesis, Cornell University, Ithaca, NY, USA, 1971. 
16. Gergely, P.; Banavalkar, P.V.; Parker, J.E. The Analysis and Behavior of Thin-Steel Hyperbolic Paraboloid Shells; A Research Project Sponsored by the America Iron and Steel Institute, Report No. 338; Civil, Architectural and Environmental Engineering: Ithaca, NY, USA, September 1971.

17. McDermott, J.F. Single layer corrugated steel sheet hypars. Proc. ASCE J. Struct. Div. 1968, 94, 1279-1294.

18. Gioncu, V.; Petcu, D. Corrugated Hypar Structures. In Proceedings of the International Conference LSCE, Warsaw, Poland, 25-29 September 1995; pp. 637-644.

19. Egger, H.; Fischer, M.; Resinger, F. Hyperschale aus Profilblechen. Stahlbau 1971, 12, 353-361.

20. Dawydov, J.U. House foods of Hyperbolic steel panel-shells. In Proceedings of the 12th International Conference on Supercomputing, ICS 1998, Melbourne, Australia, 13-17 July 1998; Volume II, pp. 500-550.

21. Davis, J.M.; Bryan, E.R. Manual of Stressed Skin Diaphragm Design; Granada Publishing Ltd.: London, UK, 1982.

22. Reichhart, A. Corrugated Deformed Steel Sheets as Material for Shells. In Proceedings of the International Conference on Lightweight Structures in Civil Engineering, Warsaw, Poland, 25-29 December 1995; pp. 625-636.

23. Prokopska, A.; Abramczyk, J. Responsive Parametric Building Free Forms Determined by Their Elastically Transformed Steel Shell Roofs Sheeting. Buildings 2019, 9, 46. [CrossRef]

24. Abramczyk, J. Transformed Shell Roof Structures as the Main Determinant in Creative Shaping Building Free Forms Sensitive to Man-Made and Natural Environments. Buildings 2019, 9, 74. [CrossRef]

25. Abramczyk, J. Symmetric Shape Transformations of Folded Shell Roofs Determining Creative and Rational Shaping of Building Free Forms. Symmetry 2019, 11, 1438. [CrossRef]

26. Biswas, M.; Iffland, J.S.B. Metal deks used to form hypar-shell panels. In Proceedings of the 2nd Speciality Conference on Cold-Formed Steel Structures, Rolla, MO, USA, 15-17 August 1973.

27. Abramczyk, J. Parametric shaping of consistent architectural forms for buildings roofed with corrugated shell sheeting. J. Archit. Civ. Eng. Environ. 2017, 10, 5-18.

28. Abramczyk, J. Principles of geometrical shaping effective shell structures forms. JCEEA 2014, XXXI, 5-21. [CrossRef]

29. Prokopska, A.; Abramczyk, J. Innovative systems of corrugated shells rationalizing the design and erection processes for free building forms. J. Archit. Civ. Eng. Environ. 2017, 29-40. [CrossRef]

30. Prokopska, A. Methodology of Architectural Design Preliminary Phases of the Architectural Process; Publishing House of Rzeszow University of Technology: Rzeszów, Poland, 2018.

31. Abramczyk, J. Integrated building forms covered with effectively transformed folded sheets. J. Int. Assoc. Shell Spat. Struct. 2016, 57, 121-132. [CrossRef]

32. Obrębski, J.B. Observations on Rational Designing of Space Structures. In Proceedings of the Symposium Montpellier Shell and Spatial Structures for Models to Realization IASS, Montpellier, France, 20-24 September 2004; pp. 24-25.

33. Rębielak, J. Morphology of roof structure systems designer by means of lenticular girder. In Spatial Structures-Temporary and Permanent, Proceedings of the International Symposium of the International Association for Shell and Spatial Structures, Shanghai, China, 8-12 November 2010; Zhang, Q., Yang, L., Hu, Y., Eds.; China Architecture \& Building Press: Shanghai, China, 2010; pp. 1249-1256.

34. Abel, J.F.; Mungan, I. Fifty Years of Progress for Shell and Spatial Structures; International Association for Shell and Spatial Structures: Madrid, Spain, 2011.

35. Dzwierzynska, J.; Prokopska, A. Pre-rationalized parametric designing of roof shells formed by repetitive modules of Catalan surfaces. Symmetry 2018, 10, 105. [CrossRef]

36. Carmo, M.P. Differential Geometry of Curves and Surfaces; Prentice-Hall, Inc.: Englewood Cliffs, NJ, USA, 1976.

37. Grey, A. Modern Differential Geometry of Curves and Surfaces with Mathematica; CRC Press LCC: Boca Raton, FL, USA, 1999.

38. Żwirek, P. Selected issues of using nominally flat folded sheets and self-bearing panels for curved. In Proceedings of the 9th Scientific and Technical Conference on Shaping Structures. Thin-walled Structures, Rzeszów, Poland, 11-12 September 2015. (In Polish).

(C) 2020 by the author. Licensee MDPI, Basel, Switzerland. This article is an open access article distributed under the terms and conditions of the Creative Commons Attribution (CC BY) license (http://creativecommons.org/licenses/by/4.0/). 